\title{
Osteotomien am proximalen Femur bei Kindern
}

\author{
Theddy F. Slongo ${ }^{1}$
}

\section{Zusammenfassung Operationsziel}

Sichere und gemäß der präoperativen Planung genaue Korrekturosteotomie des proximalen Femurendes, um eine für Bewegung und/oder Teilbelastung ausreichende Stabilität und rascher knöcherne Heilung zu erreichen.

\section{Indikationen}

Diese richten sich nach der Grundkrankheit und Deformität.

Subluxation des Femurkopfs bei verbliebener Hüftdysplasie.

Insuffizientes Containment bei Morbus Perthes.

Subluxationen bei neuromuskulären Erkrankungen.

Rotationsfehler am proximalen Femurende (Coxa antetorta/retrotorta).

Posttraumatische Fehlstellungen.

Kontraindikationen

Prinzipiell keine; das Abwägen zwischen Indikation und Kontraindikation setzt die Kenntnis der jeweiligen Krankheit und Deformität voraus.

\section{Operationstechnik}

Wir kennen die freie „Center-Center-Technik“ sowie die Technik nach präoperativer Planung.

Center-Center-Technik: Der Führungsdraht wird genau parallel zur CCD-Winkel-Linie sowie exakt im Zentrum des Schenkelhalses im axialen Strahlengang eingebracht. Die Korrektur wird durch den Schrauben/Platten-Winkel bestimmt $\left(100^{\circ} / 110^{\circ}\right.$ bzW. $\left.150^{\circ}\right)$.
Geplante/berechnete Technik: Der Führungsdraht wird entsprechend dem geplanten Korrekturwinkel und in Relation zur gewählten Platte $\left(100^{\circ} / 110^{\circ}\right.$ bzw. $\left.150^{\circ}\right)$ in der anteroposterioren Ebene sowie im Zentrum des Schenkelhalses im axialen Strahlengang platziert.

\section{Weiterbehandlung}

Je nach Alter, Grundkrankheit und gewähltem Implantat kann gipsverbandfrei, d.h. ohne Becken-Bein-Gipsverband, nachbehandelt werden: Kinder $\leq 5$ Jahre in der Regel nur im Buggy oder Rollstuhl, Kinder $>5$ Jahre Mobilisation an Gehstöcken.

\section{Ergebnisse}

Die Resultate hängen von der Erfahrung, der Genauigkeit der Planung und der Durchführung der Osteotomie ab. Korrekturverluste sind beschrieben und bekannt. Eine einwandfrei geplante Korrektur und auch komplikationslose/ gute Heilung müssen nicht immer mit einem optimalen funktionellen Ergebnis einhergehen.

\section{Schlüsselwörter}

Femurosteotomie - Proximales Femur .

Varusosteotomie Valgusosteotomie .

Derotationsosteotomie - Antetorsion - Retroversion - Zerebralparese $\cdot$ Hüftdysplasie $\cdot$ Morbus Perthes · CAPOS - LCP-Kinder-Hüftplatte $\cdot$ Intertrochantäre Osteotomie

Oper Orthop Traumatol 2008;20:334-53

DOI 10.1007/s00064-008-1406-8

\footnotetext{
${ }^{1}$ Abteilung für Pädiatrische Chirurgie, Universitätskinderklinik
} Inselspital, Bern, Schweiz. 


\section{Intertrochanteric Osteotomy of the Proximal Femur in Childhood}

\section{Abstract \\ Objective}

Intertrochanteric osteotomy of the proximal femur in a safe way and following the preoperative planning to achieve sufficient stability for free movement and partial weight bearing.

\section{Indications}

These depend on the underlying disease and the deformity. Partial dislocation of the femoral head in case of residual hip dysplasia. Loss of containment in Perthes' disease. Partial dislocation of the femoral head in neuromuscular disease.

Rotational failures of the proximal femur (coxa antetorta/ retrotorta).

Posttraumatic deformities.

\section{Contraindications}

In principle, none; the decision between indication and contraindication requires knowledge of the respective disease and deformity.

\section{Surgical Technique}

We know two different planning and surgical techniques, the so-called center-center technique and the calculated technique.

Center-center technique: the guide wire is inserted parallel and in the center of the femoral neck in anteroposterior

\section{Vorbemerkungen}

Korrekturbedürftige Fehlstellungen des proximalen Femurs können verschiedene Ursachen haben. Unter die angeborenen Ursachen fallen hauptsächlich Residualzustände nach behandelter oder unbehandelter Hüftdysplasie, angeborene Coxa ante- oder retrotorta, Pseudarthrosen des Schenkelhalses und extreme Coxa valga. Als erworbene Ursachen sind der Morbus Perthes, posttraumatische Zustände, Hüftkopffehlstellungen nach Hüftkopfgleiten sowie neuromuskuläre Erkrankungen mit Entwicklung einer sekundären Hüftdysplasie zu betrachten.

Die Indikation zur Korrektur hängt von verschiedenen Faktoren ab. Bei den residuellen Hüftdysplasien wie auch dem Morbus Perthes und neuromuskulären and axial view. The correction is determined by the screw/ plate angle $\left(100^{\circ} / 110^{\circ}\right.$ and $150^{\circ}$, respectively).

Planned/calculated technique: the guide wire is inserted in the preoperatively planned angle in relation to the chosen plate $\left(100^{\circ}, 110^{\circ}\right.$ or $\left.150^{\circ}\right)$ using the angular aiming device for the anteroposterior view; in the axial view the guide wire is also placed parallel and into the center of the femoral neck.

\section{Postoperative Management}

Depending on the child's age, the underlying disease and the chosen implant, a hip spica cast-free treatment should be striven for: children $\leq 5$ years normally in a "buggy" or wheelchair, children $>5$ years mobilization with crutches.

\section{Results}

The results depend on the experience, the accuracy of the planning and the performance of the osteotomy. Correction losses are described and reported. A faultlessly planned correction and an uncomplicated/good healing do not always have to be accompanied by an optimal functional result.

\section{Key Words \\ Femoral osteotomy · Proximal femur - Varus osteotomy · Valgus osteotomy · Derotation osteotomy · Anteversion - Retroversion - Cerebral palsy - Hip dysplasia - Perthes' disease - CAPOS system · LCP Pediatric Hip Plate · Intertrochanteric osteotomy}

Erkrankungen geht es vorwiegend um eine Verbesserung der Hüftkopfüberdachung, das sog. Containment. Bei der Coxa ante- oder retrotorta hingegen können durchaus auch Gangprobleme oder gar ästhetische Beeinträchtigungen Grund für die Korrektur sein. Solche Korrekturen sollten nur von erfahrenen, kinderorthopädisch geschulten Chirurgen indiziert und durchgeführt werden.

Heute stehen dafür prinzipiell zwei Operationstechniken zur Verfügung:

1. Die weltweit seit Jahrzehnten bekannte Technik mit einer Winkelplatte. Diese recht schwierige Methode konnte vor kurzem durch die Einführung kanülierter Meißel deutlich vereinfacht und sicherer gemacht werden (CAPOS-System, Fa. Synthes). 
2. Das seit 2007 erhältliche LCP-Pädiatrie-Hüftplattensystem (Fa. Synthes), das eine winkelstabile Fixierung ohne Verwendung des problematischen PlattenmeiBels erlaubt. Diese Technik ermöglicht aufgrund ihrer hohen Stabilität auch eine gipsverbandfreie Nachbehandlung.

Die Winkelplattentechnik dürfte künftig durch die weniger aufwendige und präzisere Operationstechnik mit Kinder-Hüftplatten abgelöst werden (Abbildung 1).

\section{Operationsprinzip und -ziel}

Herstellung normaler Winkelverhältnisse zwischen Femurschaft und Femurhals sowie einer korrekten Antetorsion des proximalen Femurs durch eine interbis subtrochantäre Osteotomie. Bessere Einstellung

\section{Vorteile}

\section{Allgemein}

- Sowohl die Winkelplatte als auch die LCP-Kinder-Hüftplatte erlauben - im Gegensatz zur Kirschner-Draht- oder Schraubenfixation - eine geplante Korrektur und vermögen diese auch stabil zu halten.

\section{Cannulated Pediatric Osteotomy System (CAPOS)}

- Sicherere Platzierung des Plattensitzinstruments durch Verwendung eines Ziel-/Führungsdrahts. Dadurch im Vergleich zum herkömmlichen System Verringerung der Gefahr einer Plattenfehllage und Perforation des Schenkelhalses mit dem Meißel und einer anschließenden Durchblutungsstörung des Hüftkopfs.

- Enorme Vielseitigkeit durch verschiedene Plattengrößen sowie entsprechende Klingenlängen.

- Vom 2. Lebensjahr bis zur Adoleszenz anwendbar.

- Funktionelle Nachbehandlung möglich.

- Durch Verwendung einer sog. Kalkarschraube (nur auf Anfrage erhältlich) hohe Ausrissfestigkeit auch bei osteoporotischem Knochen, wie z.B. bei Patienten mit Zerebralparese oder anderen neuromuskulären Erkrankungen.

\author{
Abbildung 1 \\ Neue winkel- \\ stabile \\ LCP-Kinder- \\ Hüftplatte \\ (System Fa. \\ Synthes). Sie \\ zeichnet sich \\ durch eine \\ sehr hohe Fes- \\ tigkeit aus, vor \\ allem auf- \\ grund der Ver- \\ wendung der \\ sog. Kalkar- \\ schraube.
}

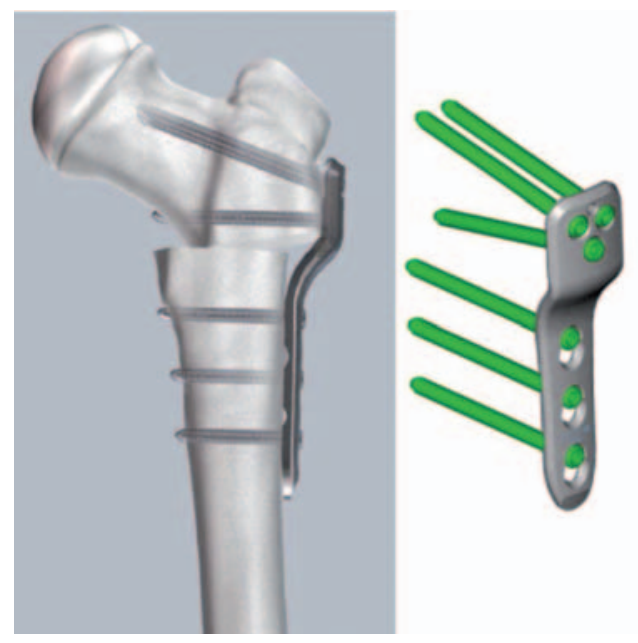

der Hüfte (Containment) durch eine Varusosteotomie bei ungenügender Überdachung des Femurkopfs. Stabile Osteosynthese der Osteotomie mit der Möglichkeit einer funktionellen Nachbehandlung ohne Korrekturverlust.

\section{LCP-Kinder-Hüftplatte}

- Das anatomische Plattendesign erleichtert die Platzierung der Platte am proximalen Femur.

- Minimiert die Irritation der Weichteile, wie man sie bei der Winkelplatte sieht.

- Die Winkelstabilität reduziert das Risiko eines primären oder sekundären Korrekturverlusts.

- Der minimale Knochenkontakt zwischen Platte und Knochen kompromittiert die periostale Zirkulation nicht.

- Vereinfacht die Operationstechnik.

- Erlaubt intraoperative Korrekturen ohne Stabilitätsverlust.

- Durch die Vielzahl der verschiedenen Schraubenlängen ist eine bessere Anpassung an die Länge des Schenkelhalses als mit der Klinge möglich.

- Nur zwei Plattengrößen.

- Drei verschiedene Plattentypen (Varus $100^{\circ} / 110^{\circ}$, Valgus $150^{\circ}$ ).

- Gipsverbandfreie Nachbehandlung.

\section{Nachteile Beide Systeme}

- Relativ großer Eingriff.

- Relativ mächtiges Implantat. 
- Zweiter großer Eingriff zur Materialentfernung notwendig.

- Implantatkosten, besonders bei LCP-Kinder-Hüftplatte.

\section{Indikationen}

- Die Indikationen für eine Umstellung am proximalen Femur hängen von verschiedenen Faktoren und Gegebenheiten ab.

- Alter: Bei jüngeren Kinder ist nach der Umstellung noch eine weitere positive Entwicklung zu erwarten, d.h., das junge Alter wirkt sich positiv auf ein Remodeling nach Umstellung aus. Bezüglich des Alters ist auch die Frage nach irreversibler Schädigung oder negativer Entwicklung in Betracht zu ziehen. Der bisherige Verlauf ist ebenfalls von größter Wichtigkeit. Folgende Altersgruppen sind dabei zu unterscheiden: Kleinkind, Vorschulkind (sog. Toddler), Schulkind und Adoleszenten.

- Subluxation bei Patienten mit Zerebralparese und Morbus Perthes; die Wiederherstellung des sog. Containments ist hier anzustreben.

- Subluxationen bei residueller Hüftdysplasie.

- Rotationsfehler, sog. Coxa ante- oder retrotorta; die Indikation ist mit dem Patienten respektive den Eltern zu diskutieren: Wird die Korrektur aus kosmetischen oder sozialen Gründe (Angst, ausgelacht zu werden) gewünscht?

- Coxa vara congenita/Coxa valga.

\section{Kontraindikationen}

- Keine.

- Schwere Dysplasien des Azetabulums sollten besser durch eine Beckenosteotomie, evtl. in Kombination mit einer Femurosteotomie, korrigiert werden.

\section{Patientenaufklärung}

- Bei einer Fraktur können Eltern Therapievorschläge meist ohne Weiteres nachvollziehen. Anders verhält es sich bei Korrektureingriffen; hier verstehen die Eltern die ärztlichen Überlegungen oft nur schwer. Es ist deshalb von größter Wichtigkeit, einerseits über die Indikation gut verständlich aufzuklären, andererseits auch über den Operationsverlauf und die Nachbehandlung sowie die möglichen Folgen klar Auskunft zu geben.

\section{Aufklärung bezüglich Indikation}

- Erklärung der Bedeutung einer nicht zentrierten Hüfte sowie der Möglichkeit von Luxation, Schmerzen und Früharthrose.
- Erklärung anerkannter Kriterien für eine Korrekturindikation.

- Auswirkung des Zeitfaktors hinsichtlich der Korrektur: Jüngere Kinder haben mehr Möglichkeiten des Remodelings, die Pfanne adaptiert sich besser an den Hüftkopf.

- Erklärung radiologischer Beispiele einer normalen Hüfte im Vergleich zur Hüfte des erkrankten Kinds.

- Besonders bei Patienten mit Zerebralparese Hinweis zu Schmerzen und pflegerischen Problemen.

\section{Aufklärung bezüglich Operation (unabhängig von der gewählten Methode)}

- Allgemeine chirurgische Risiken.

- Schnittführung.

- Knochendurchtrennung und Fixierung.

- Art der Korrektur.

- Operationsziel.

- Spezifische Komplikationen wie Schrauben-, Klingen- oder Plattenbruch.

- Plattenausriss besonders bei ostopenischem Knochen.

- Geplante Implantatentfernung.

- Hospitalisationsdauer.

- Zusätzliche Fixation, z.B. mit Becken-Bein-Gipsverband.

\section{Aufklärung bezüglich Nachbetreuung und Resultat}

- Möglicher Korrekturverlust wegen ungenügender Fixation.

- Korrekturverlust aufgrund des Wachstums.

- Freies Gehen ab Heilung.

- Besonders bei Derotationen kann nicht immer vorausgesagt werden, wie das Kind mit der neuen Situation zurechtkommt.

\section{Operationsvorbereitungen}

- Übliche Laborabklärungen wie Hämoglobin, Leukozyten, Senkung, Blutgruppe, in Ausnahmefällen auch Quick.

- Bereitstellung von Blutkonserven nicht notwendig.

- Aktuelles präoperatives Röntgenbild, nicht älter als 6 Monate.

- Funktionelle Röntgenaufnahme in maximaler Abduktion und $25^{\circ}$ Innenrotation.

- Sicherstellung der intraoperativen Durchleuchtungsmöglichkeit für Operation in Rücken- und Seitenlage.

\section{Präoperative Planung}

- Wesentlicher Bestandteil einer intertrochantären Osteotomie. 

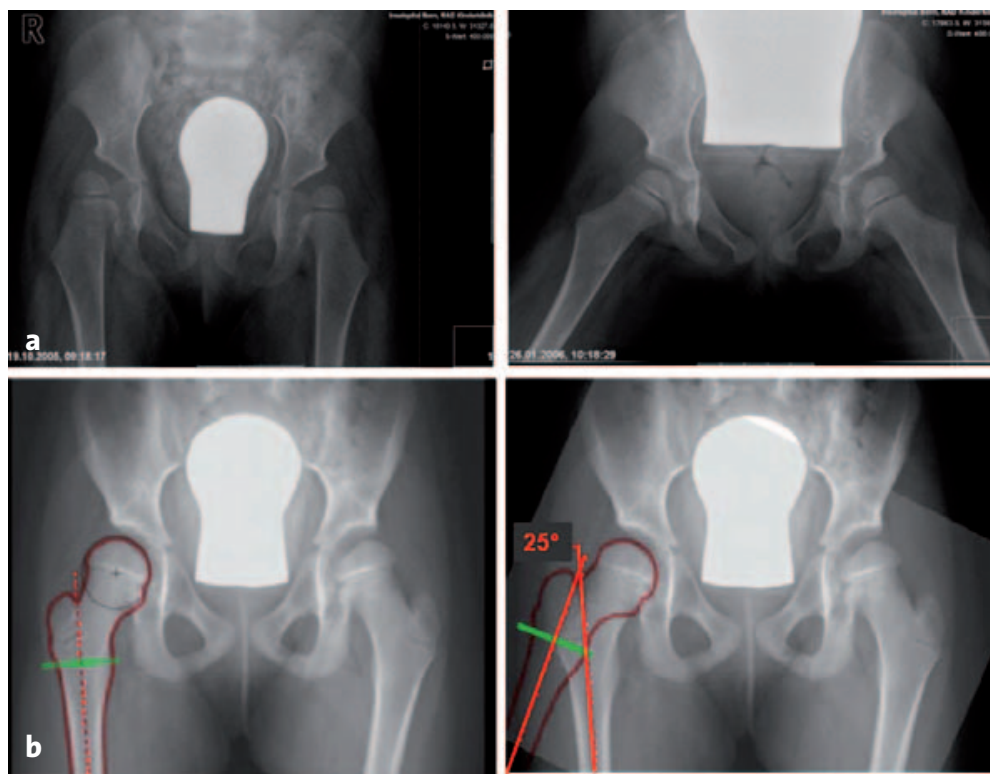

\section{Abbildungen $2 a$ und $2 b$}

a) Korrekte a.p. Röntgenaufnahmen des Beckens, die erste mit parallelen Beinen und $25^{\circ}$ nach innen gedrehten Füßen, die zweite ebenfalls mit $25^{\circ}$ nach innen gedrehten Füßen und so weit abduziert, dass eine optimale Zentrierung der Hüfte erreicht wird.

b) Auf beiden Bildern wird die Femurlängsache eingezeichnet, und die Bilder werden übereinander projiziert. Der Winkel zwischen den beiden Längsachsen entspricht dem Korrekturwinkel.

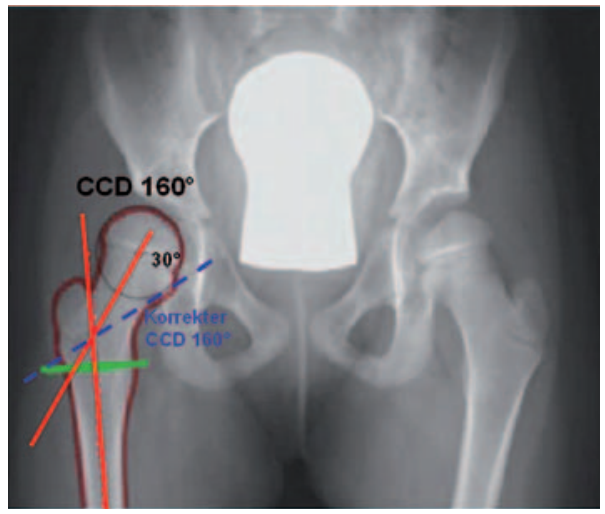

\section{Abbildung 3}

Planung, ausgehend vom Schenkelhalswinkel: Ausgangsreferenz bei dieser Planung ist der aktuelle Schenkelhals-Diaphysen-(CCD-) Winkel. Ziel ist ein möglichst normaler oder optimaler CCD-Winkel, in der Regel zwischen $130^{\circ}$ und $120^{\circ}$.
- Basis stellt die funktionelle Aufnahme (Operationsvorbereitung) dar.

- Ziel ist die Berechnung der Korrektur, um eine möglichst anatomiegerechte Situation am proximalen $\mathrm{Fe}$ mur und eine optimale Zentrierung des Hüftkopfs im Azetabulum zu erreichen.

- Ausnahme: Korrektur bei Subluxationen und sehr dysplastischem Azetabulum bei Kindern mit neuromuskulären Erkrankungen. Hier strebt man eine maximale Korrektur mit der sog. Center-Center-Technik bis zu einem Schenkelhals-Diaphysen-(CCD-)Winkel von $90^{\circ}$ an. Der Korrekturwinkel wird meist durch die entsprechende Platte vorgegeben.

- Korrekturplanung einer varisierenden Osteotomie mit Hilfe der Schaftachse, „funktionelle Planung“:

- Die Basis dieser Planung stellen zwei korrekte anteroposteriore (a.p.) Beckenübersichtsaufnahmen dar, die erste mit parallelen Beinen und $25^{\circ}$ nach innen gedrehten Füßen, die zweite ebenfalls mit $25^{\circ}$ nach innen gedrehten Füßen und so weit abduziert, dass eine optimale Zentrierung der Hüfte erreicht wird.
- Auf beiden Bildern Einzeichnen der Femurlängsachse.

- Übereinanderprojektion der Bilder.

- Der Winkel zwischen den beiden Längsachsen entspricht dem Korrekturwinkel.

- Will man auf die zweite Aufnahme verzichten, kann eine Pause der Beckenübersichtsaufnahme angefertigt und so weit um das Femurkopfzentrum gedreht werden, bis die gewünschte Kopfzentrierung erreicht ist: Dieser Drehwinkel zwischen den Längsachsen ergibt ebenfalls den Korrekturwinkel (Abbildungen 2a und 2b).

- Korrekturplanung einer varisierenden Osteotomie, ausgehend vom Schenkelhalswinkel:

- Referenz bei dieser Planung ist der aktuelle CCD-Winkel.

- Ziel ist ein möglichst normaler CCD-Winkel, in der Regel zwischen $130^{\circ}$ und $120^{\circ}$.

- Der Korrekturwinkel ergibt sich aus der Differenz des aktuellen und des geplanten CCD-Winkels; z.B. aktuell $165^{\circ}$ Valgus, Planung $125^{\circ}$ entspricht einer $40^{\circ}$-Varuskorrektur (Abbildung 3 ). 


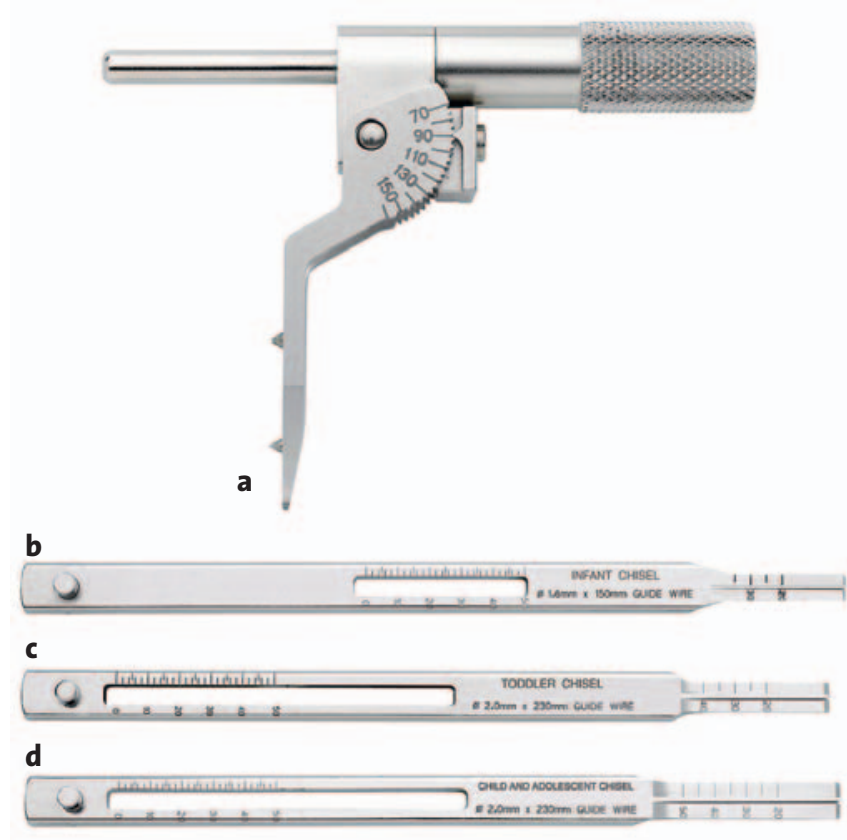

\section{Abbildungen $4 \mathrm{a}$ bis $4 \mathrm{f}$}

Originalinstrumente (Fa. Synthes) für die Applikation einer Winkelplatte mit dem kanülierten Plattensitzinstrument. a) Justierbares Zielgerät zur Platzierung des Kirschner-Führungsdrahts.

- Entsprechende Planung für valgisierende Osteotomien von Varus in Valgus und zum normalen CCD-Winkel: Für die vom Schaftwinkel ausgehende Planung wird das Bein maximal adduziert; der Korrekturwinkel entspricht der Differenz von Neutral- und Adduktionsstellung.

- Rotationskorrekturen werden meist in Kombination mit Varuskorrekturen durchgeführt, da sich durch die Veränderung der Rotation der Schenkelhalswinkel mit verändert! Dies muss während der Operation funktionell wie radiologisch überprüft werden. Die Abduktionsfähigkeit darf nicht vermindert werden.

\section{Instrumentarium und Implantate}

- Prinzipiell können proximale Femurosteotomien mit verschiedenen Implantaten fixiert werden. Für ein optimales Resultat empfehlen wir, die speziell für diese Operation vorgesehenen Instrumente und Implantate zu verwenden. Sie sind so ausgelegt, dass einerseits sicher operiert werden kann, andererseits eine übungsstabile Fixierung erreicht wird.

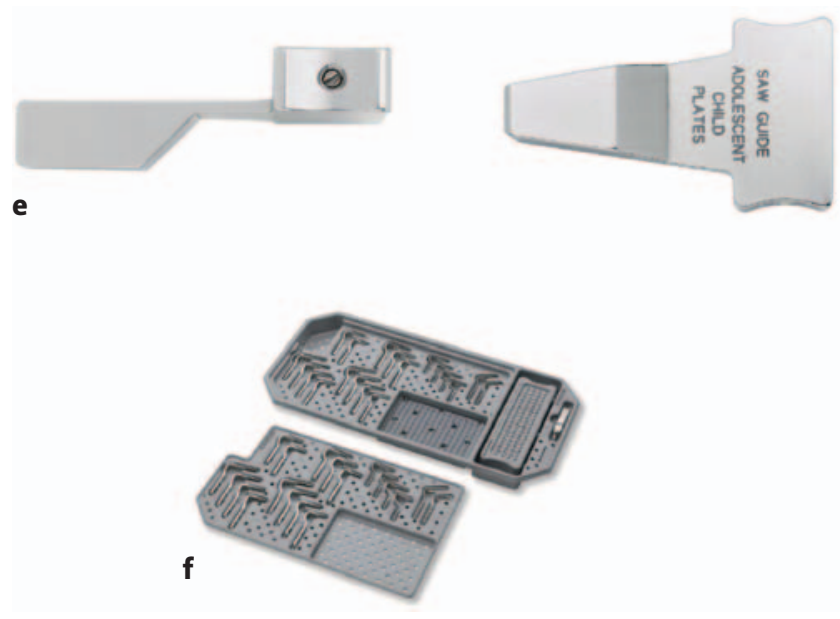

b-d) Kanülierte Plattensitzinstrumente für Kleinkinder (b), Kinder (sog. Toddler; c) und Adoleszenten (d).

e) Sägeblock entsprechend der Meißel- bzw. Plattengröße. f) Winkelplatten verschiedener Größen für Kleinkinder, Vorschulkinder, Kinder und Adoleszenten.

- Übliche orthopädisch-traumatologische Grundinstrumente.

- Standardinstrumente CAPOS (Cannulated Pediatric Osteotomy System, Synthes $\mathrm{GmbH}$ ):

- Justierbares Zielgerät mit Winkeleinteilung für Führungsdraht (Abbildung 4a).

- Kanüliertes Plattensitzinstrument für Kleinkinder-, Kinder- und Adoleszentenplatte (Abbildungen $4 \mathrm{~b}$ bis $4 d$ ).

- Sägeblock entsprechend der Plattenknietiefe (Abbildung 4e).

- Winkelplatten für Kleinkinder, Kinder und Adoleszenten mit verschiedenen Klingenlängen und unterschiedlichem Offset (Abbildung 4f).

- Standardinstrumente LCP-Kinder-Hüftplatte (Synthes $\mathrm{GmbH}$ ):

- Zielplatten, dreieckig, $45 \mathrm{~mm}, 90^{\circ} / 50^{\circ} / 40^{\circ}$, $80^{\circ} / 70^{\circ} / 30^{\circ}$ und $100^{\circ} / 60^{\circ} / 20^{\circ}$ (Abbildung 5a).

- Griff für Drehmomentbegrenzer (Abbildung 5b).

- Drehmomentbegrenzer, 1,5 Nm bei 3,5-mm-Platte und $4 \mathrm{Nm}$ bei 5,0-mm-Platte (Abbildung $5 \mathrm{c}$ ). 

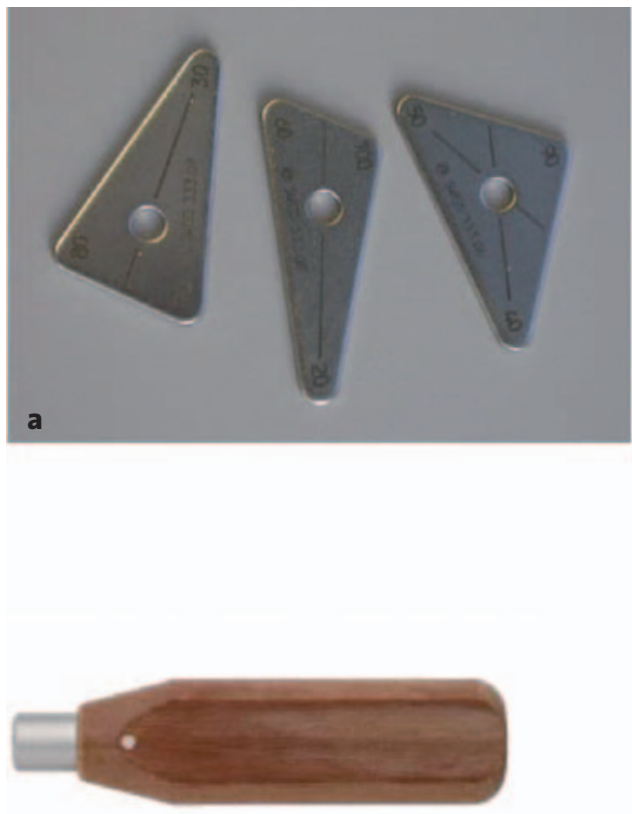

b
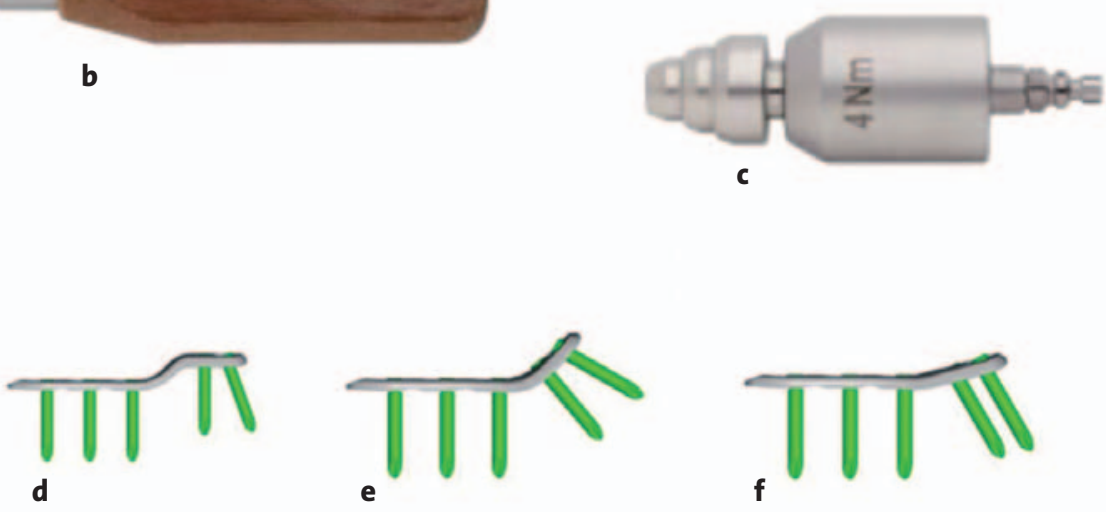
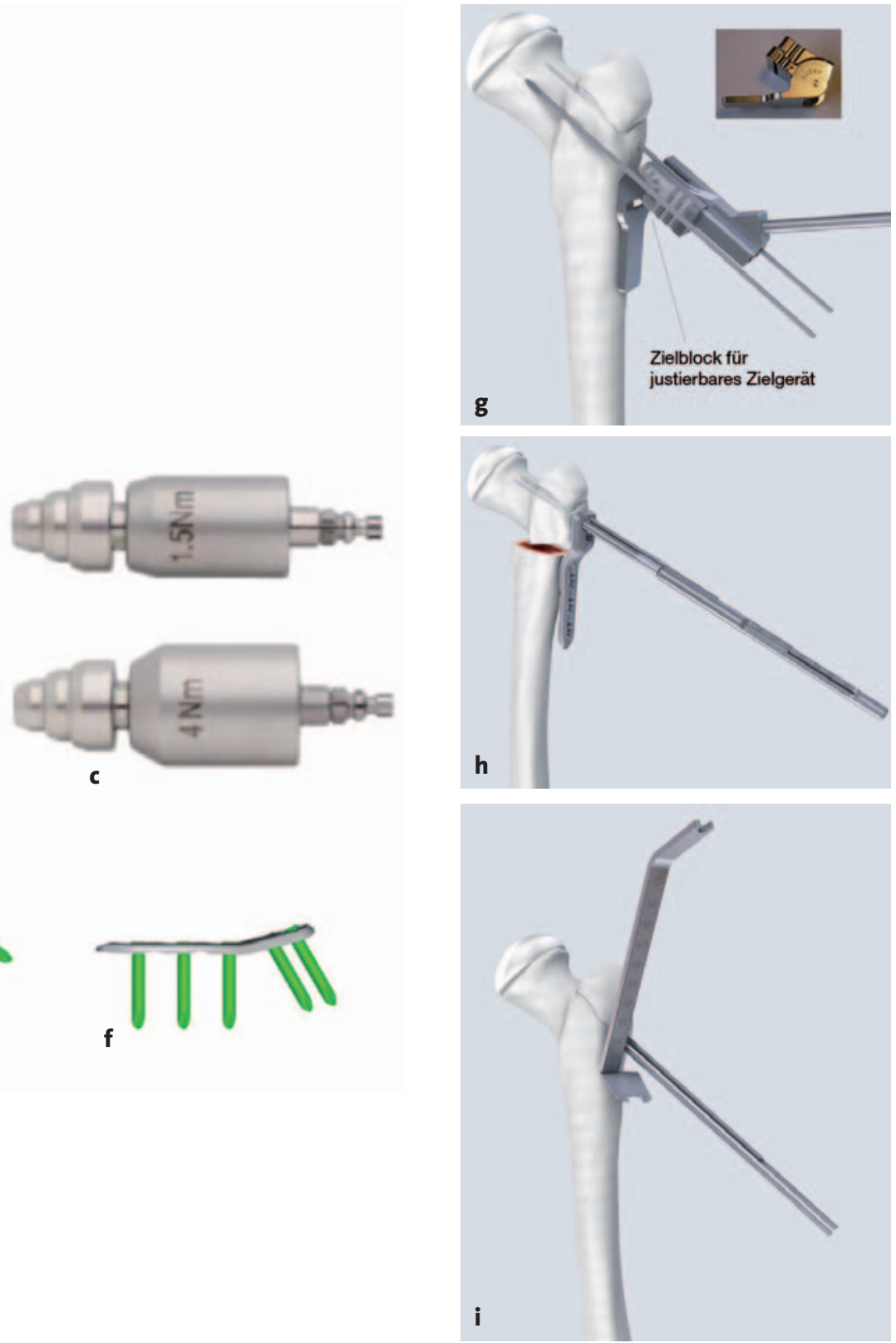

\section{Abbildungen 5 a bis $5 \mathbf{j}$}

Originalinstrumente (Fa. Synthes) für die neue LCP-Kinder-Hüftplatte.

a) Zielplatten, dreieckig, $45 \mathrm{~mm}, 90^{\circ} / 50^{\circ} / 40^{\circ}, 80^{\circ} / 70^{\circ} / 30^{\circ}$ respektive $100^{\circ} / 60^{\circ} / 20^{\circ}$.

b) Griff für Drehmomentbegrenzer.

c) Drehmomentbegrenzer, 1,5 $\mathrm{Nm}$ bei 3,5- $\mathrm{mm}$-Platte respektive $4 \mathrm{Nm}$ bei 5,0-mm-

Platte.

d) Varusplatte, $3,5 \mathrm{~mm}, 100^{\circ}$ respektive $110^{\circ}$ (5,0 mm identische Winkel).

e) Valgusplatte, $3,5 \mathrm{~mm}, 150^{\circ}$ (5,0 $\mathrm{mm}$ identischer Winkel).

f) Frakturplatte, $3,5 \mathrm{~mm}, 120^{\circ}$ (5,0 $\mathrm{mm}$ identischer Winkel).

g) Zielblock für justierbares Zielgerät und Schraubeninstrumente. Justierbares Zielgerät mit Winkeleinteilung.

h) Messstab für Kirschner-Drähte, Instrument 2,8 mm, Länge $200 \mathrm{~mm}$.

i) Positionierhilfe für Osteotomie.

j) LCP-Bohrbüchse für 3,5-mm- und 5,0-mm-LCP-Platten.

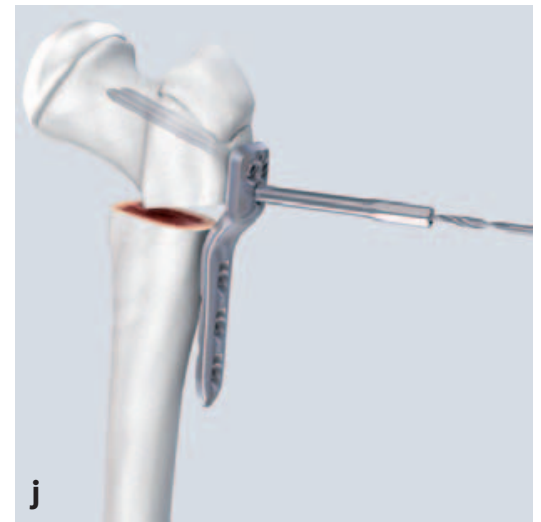


- Varusplatte, $3,5 \mathrm{~mm}, 100^{\circ}$ und $110^{\circ}$ (5,0 $\mathrm{mm}$ identische Winkel, Abbildung 5d).

- Valgusplatte $3,5 \mathrm{~mm}, 150^{\circ}$ (5,0 mm identischer Winkel, Abbildung 5e).

- Frakturplatte, $3,5 \mathrm{~mm}, 120^{\circ}(5,0 \mathrm{~mm}$ identischer Winkel, Abbildung 5f).

- Zielblock für justierbares Zielgerät undn Schraubeninstrumente (Abbildung 5g, je eines für 3,5-mmund 5,0-mm-Hüftplatten) sowie justierbares Zielgerät mit Winkeleinteilung.

- Messstab für Kirschner-Drähte, Instrument 2,8 mm, Länge $200 \mathrm{~mm}$ (Abbildung 5h).

- Positionierhilfe für Osteotomie (Abbildung 5i).

- LCP-Bohrbüchse für 3,5-mm- und 5,0-mm-LCPPlatten (Abbildung 5j).

\section{Anästhesie und Lagerung}

- Intubationsnarkose mit Relaxation; erleichtert das Manipulieren der Fragmente nach Osteotomie.

- Rückenlage (Abbildung 6):

- Der röntgenstrahlendurchlässigen Operationstisch wird so auslegt, dass eine freie Durchleuchtung möglich ist.

- Unterlage unter Rücken/Steißbein, um das Gesäß etwas anzuheben; dies erleichtert die Präparation, da die Weichteile nicht nach oben gedrückt werden.
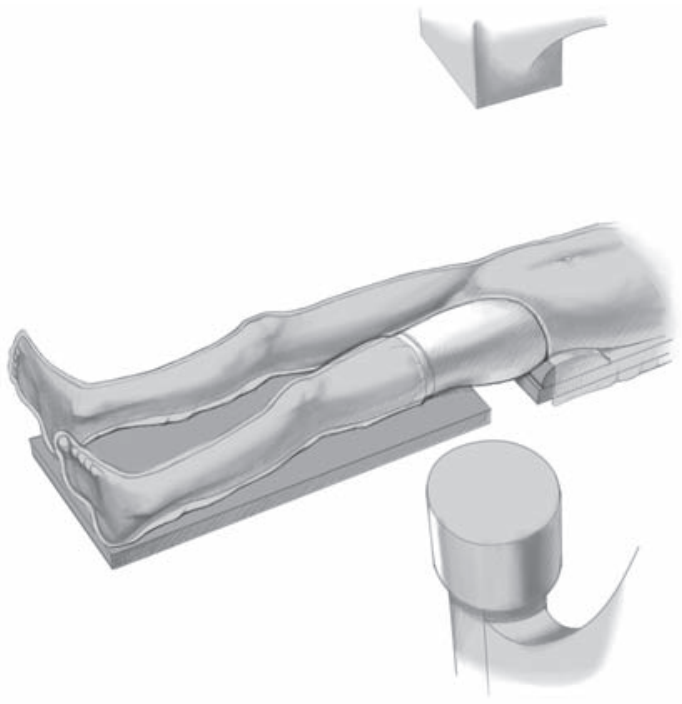

\section{Abbildung 6}

In Rückenlage des Patienten röntgenstrahlendurchlässigen Operationstisch so auslegen, dass eine freie Durchleuchtung möglich ist. Bein frei beweglich bis zum Beckenkamm abdecken.
- Die Füße des Kinds werden ganz an das Tischende gezogen.

- Desinfektion des ganzen Beins inklusive Fuß.

- Frei bewegliche Abdeckung des Beins bis zum Beckenkamm.

- Seitenlage (Abbildung 7):

- Für beide Operationstechniken empfehlen wir die Seitenlage. Sie ist noch wenig verbreitet, bietet aber operationstechnisch und bezüglich der Übersicht viele Vorteile.

- Normaler Operationstisch.

- Strenge Seitenlage.

- Der Patient wird an Rücken und Bauch mit Seitenstützen fixiert oder auf eine Vakuummatratze gelegt.

- Lagerung des Arms auf Armbank, so dass kein Zug an der Schulter und am Plexus brachialis entsteht.

- Anwinkelung des unten liegenden Beins.

- Lagerung des oben liegenden Bein auf gepolsterten, stabilen Block.

- Sterile und frei bewegliche Abdeckung des ganzen Beins bis zum Beckenkamm.

- Horizontale Einstellung des Durchleuchtungsgeräts.

- Intraoperative Durchleuchtung:

- Einstellen des Bildverstärkers auf Impulsbestrahlung.

- Nur einzelne kurze Bildsequenzen, Vermeidung des sog. Bleifußes!

- Die Durchleuchtungszeit sollte so gering wie möglich gehalten werden.

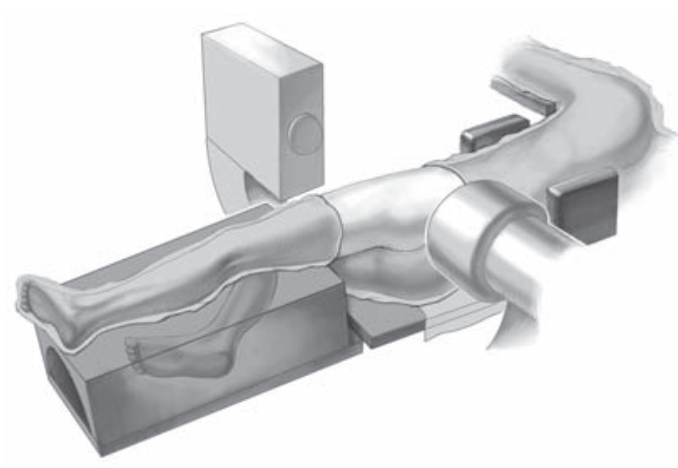

\section{Abbildung 7}

Patient auf die nicht zu operierende Seite lagern. Oben liegendes Bein auf gepolsterten, stabilen Block legen. Durchleuchtungsgerät horizontal einstellen. 


\section{Operationstechnik}

\section{Abbildungen 8 bis 21}

\section{CAPOS (Abbildungen 8 bis 15)}

Ausgangslage: Kind $8 \mathrm{Jahre}, 28 \mathrm{~kg}$, Femur mit $160^{\circ}$ Valgus; $55^{\circ}$ Anteversion.

Operation: Varisation und Derotation linkes Femur von jeweils $30^{\circ}$.

a

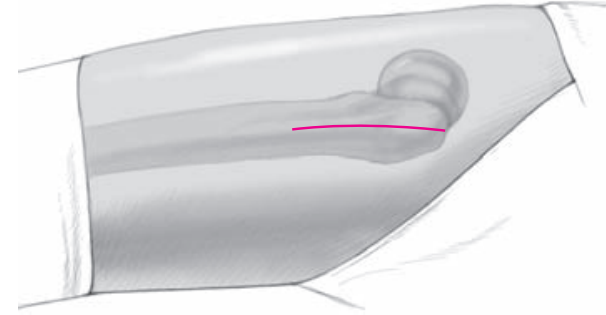

b

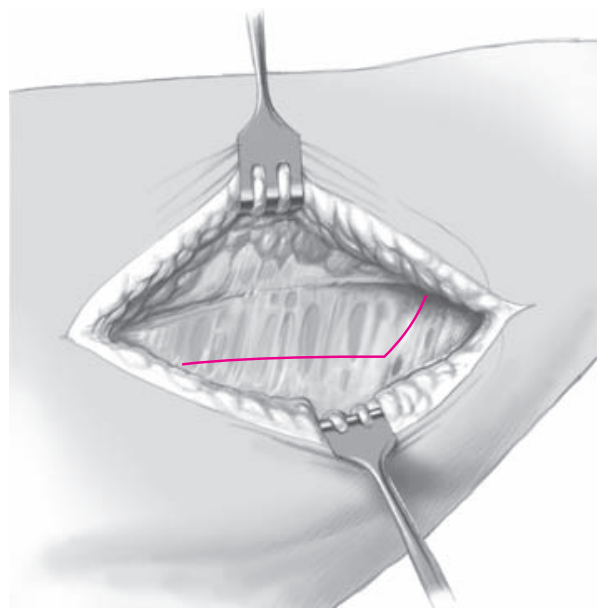

\section{Abbildungen $8 \mathrm{a}$ bis $8 \mathrm{c}$}

Hautinzision streng lateral und gerade, der Größe des Kinds angepasst und ca. 11-15 cm lang, beginnend knapp unterhalb der palpablen Trochanterspitze nach distal (a). Darstellung der Faszie und ebenfalls gerade Inzision entlang der Faserrichtung. Das Fettgewebe der Bursa trochanterica und über dem Musculus vastus lateralis wird von ventral nach dorsal abpräpariert. Der Vastusansatz wird klar sichtbar.

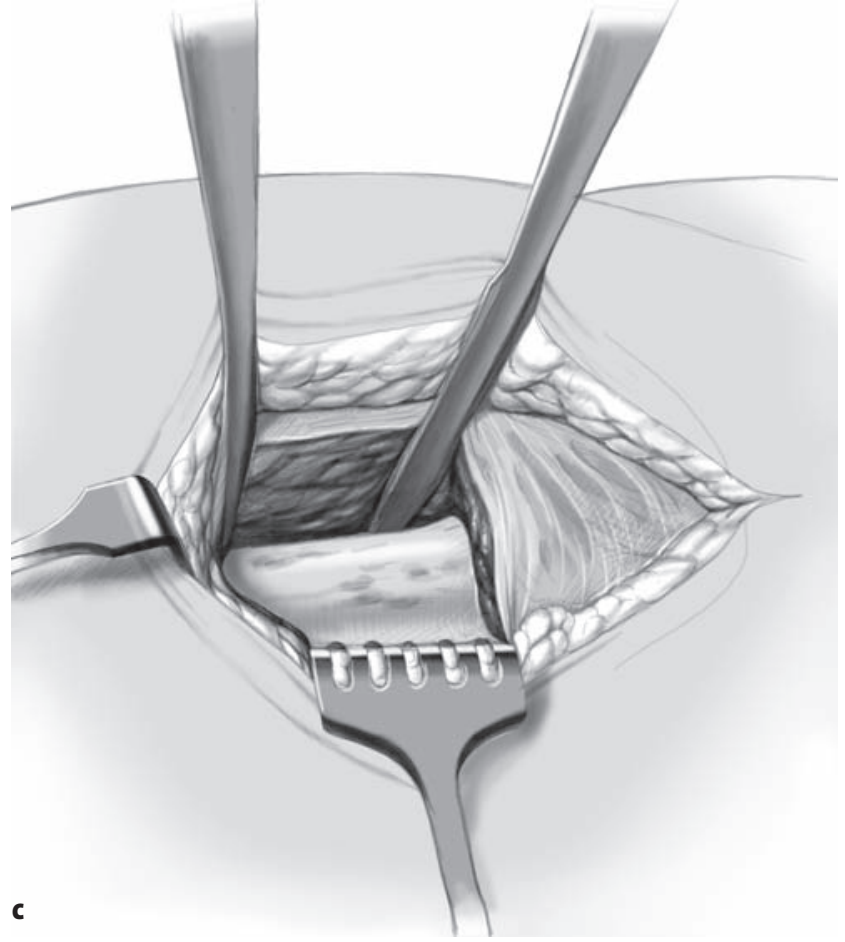

L-förmige Inzison ca. 2-3 mm unterhalb des Ansatzes bis auf den Knochen, streng dorsale Inzision des Periosts entlang der dorsalen Muskelinsertion und ventral der Insertion der Sehne des Musculus gluteus maximus (b).

Abheben des Muskels mit dem Periost, so dass das ganze proximale Femur klar zu sehen ist. Einsetzen eines stumpfen Hohmann-Retraktors ventral und dorsal auf Höhe der Intertrochantärregion, so dass das Periost hier zirkulär abgelöst ist (c). 

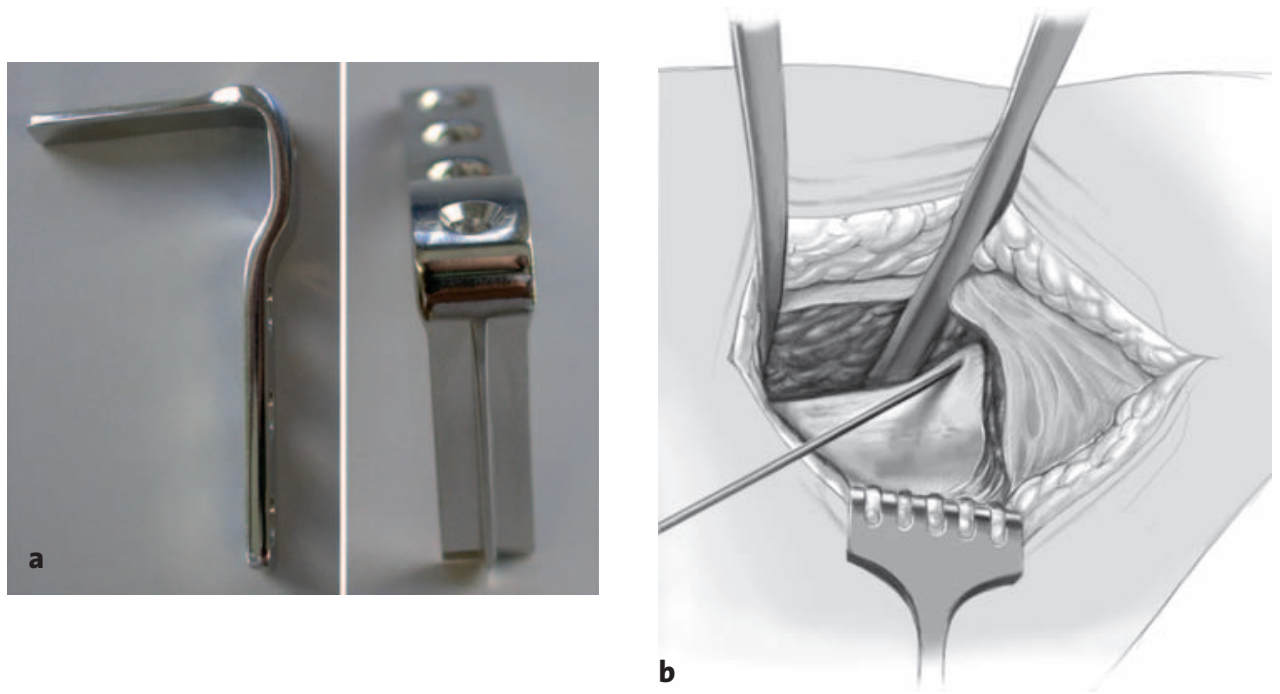

\section{Abbildungen 9a bis 9d \\ Festlegung der Plattenposition und des Plattentyps.}

Aufgrund der starken Valgusdeformität und des Alters wählen wir eine 3,5- $\mathrm{mm}$-Kinder-Hüftplatte (sog. Toddler-Platte), $90^{\circ}$ mit T-Profil, $32 \mathrm{~mm}$ Klingenlänge und $8 \mathrm{~mm}$ Offset zur Medialisierung (a).

Die künftige Plattenposition hängt von der genauen Platzierung des Kirschner-Führungsdrahts ab. Der Einführungspunkt liegt ca. $10 \mathrm{~mm}$ unterhalb der Trochanterfuge.

Setzen des Kirschner-Führungsdrahts.

Setzen des sog. Kirschner-Anteversionsdrahts auf der ventralen Fläche des Schenkelhalses und leichtes Einhämmern in den Kopf. Identifikation der Trochanterfuge und Aufsetzen des justierbaren Zielgeräts $\left(90^{\circ}+30^{\circ}=120^{\circ}\right)$ für den zentralen Führungsdraht ca. $10 \mathrm{~mm}$ unterhalb der Fuge; der Führungsdraht muss in der Frontalebene parallel zum Anteversionsdraht liegen (c). Einbohren des Kirschner-Führungsdrahts mit Hilfe des Zielgeräts; dabei muss der Schuh des Zielgeräts am Femur aufliegen. Kontrolle der Lage des Kirschner-Führungsdrahts in a.p. und axialer Durchleuchtung (d). Im axialen Bild muss der Draht im Zentrum des Schenkelhalses liegen. Die Drahtspitze sollte die Femurkopffuge nicht perforieren. Die Einschlagtiefe des Plattensitzinstruments kann später indirekt am Überstand des Führungsdrahts abgelesen werden. $0 \mathrm{~mm}$ bedeutet, dass die Klingenspitze die Spitze des Kirschner-Drahts erreicht hat.

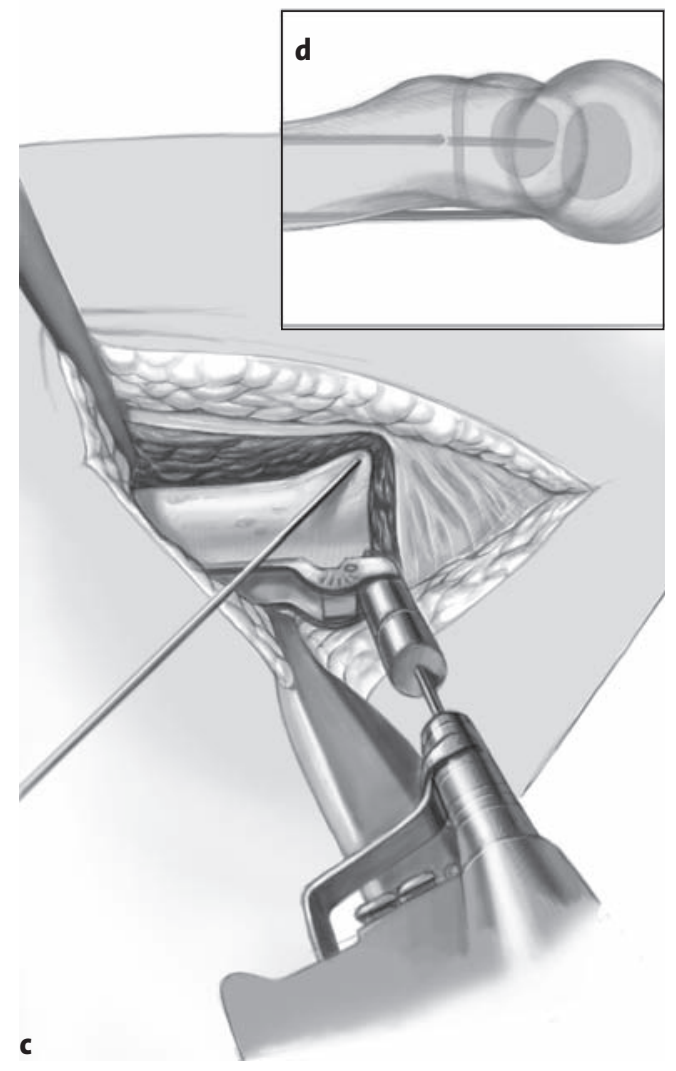



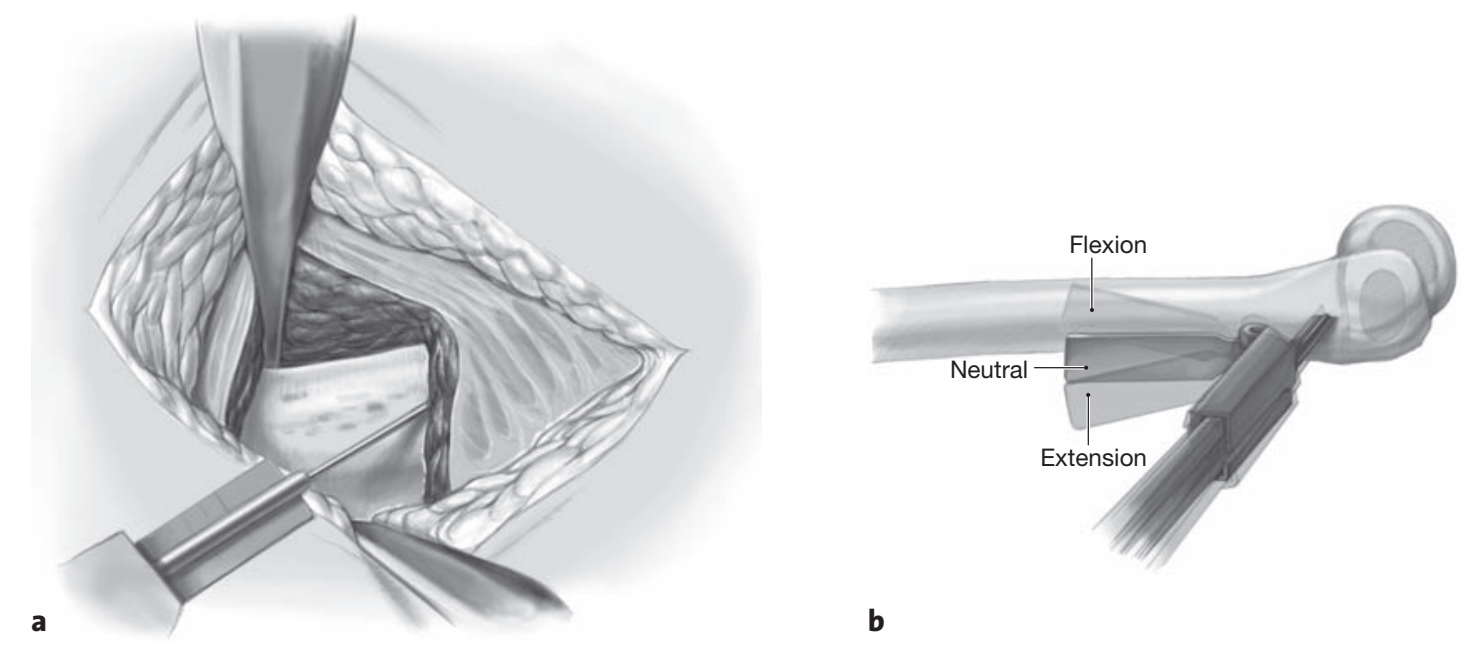

\section{Abbildungen 10a bis 10c}

Einschlagen des Plattensitzinstruments.

Das entsprechende kanülierte Plattensitzinstrument wird über den Kirschner-Führungsdraht geschoben, wobei wir darauf achten, dass die Klinge leicht gegen den Draht gedrückt wird, um ein Divergieren zu vermeiden (a).

Das Plattenführungsinstrument wird über den Plattensitz geschoben; dies dient zur Ausrichtung der Platte: Neutral, Flexion oder Extension (b).

Einschlagen des Plattensitzinstruments entlang dem Führungsdraht. Es muss darauf geachtet werden, dass mindestens nach $2 \mathrm{~cm}$ die Klinge mit Schlitzhammer immer wieder gelockert, d.h. etwas zurückgeschlagen wird (c).

Vorschlagen der Klinge bis auf die gewünschte Tiefe; diese kann am Führungsdraht in Relation zu dessen Spitze oder mit dem Bildverstärker kontrolliert werden.

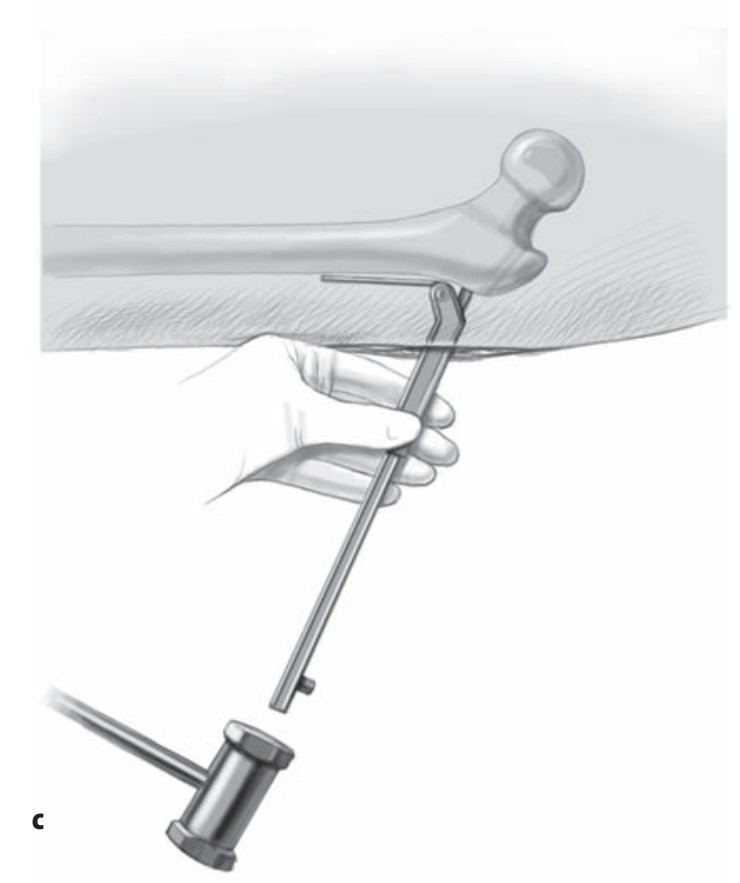




\section{Abbildung 11}

Festlegen der Osteotomiehöhe und der Rotation.

Der zur Platte korrespondierende Sägeblock wird über den Meißel des Plattensitzinstruments geschoben. Die Dicke des Blocks entspricht der Länge des Plattenknies. Setzen einer kleinen Sägemarke. Oberhalb und unterhalb dieser Marke kann nun je ein 2,0-mm-Kirschner-Draht so platziert werden, dass die Drähte entweder parallel zueinander liegen oder im Fall der geplanten Derotation in einem Winkel von $30^{\circ}$ zueinander eingebracht werden. Nach der Derotation sollten sie dann parallel stehen.

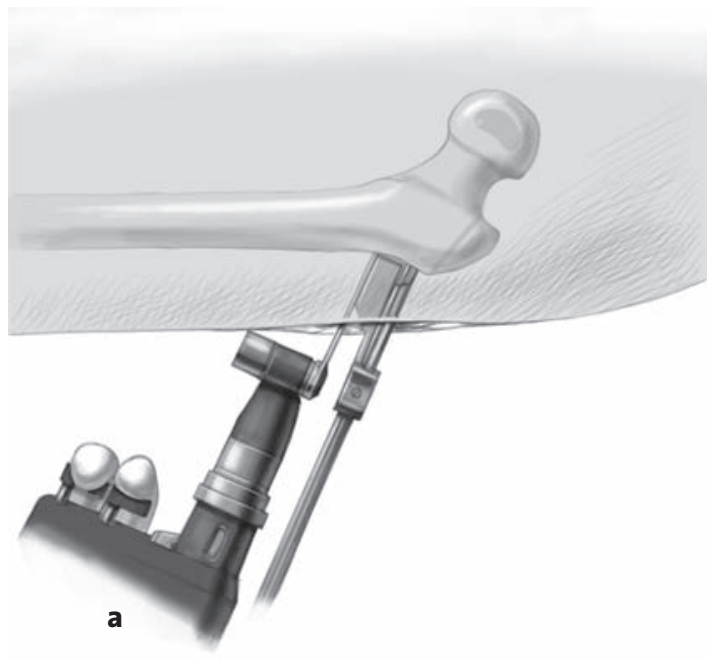

Abbildungen 12a bis 12c

Setzen der Osteotomie.

Prinzipiell stehen zwei Optionen zur Verfügung: Eine Osteotomie mit Hilfe des Sägeblocks parallel zum Plattensitzinstrument hat den Nachteil einer starken Verkürzung sowie einer dünnen Knochenbrücke zwischen Plattenklinge und Osteotomie. Zusätzlich muss ein Knochenkeil am distalen Fragment entfernt werden. Der Vorteil liegt in der großen Knochenkontaktfläche (a). Alternativ kann auch auf Höhe der gesetzten Marke rechtwinklig zum Schaft osteotomiert werden. Der Nachteil liegt hier in der kleineren Knochenkontaktfläche. Die Vorteile bestehen in der geringeren Verkürzung sowie der kräftigen Knochenbrücke. Der Knochen wird mit einem breiten Sägeblatt durchtrennt; die Weichteile schützen wir mit zwei stumpfen, breiten Hohmann-Haken (b). Die Osteotomie wird an der medialen Kortikalis nicht vollständig ausgeführt, um die Gefäße zu schützen. Mit einem breiten Meißel im Osteotomiespalt und maximaler Abduktion des Beins bricht man den Knochen vollständig und löst das Periost (c).
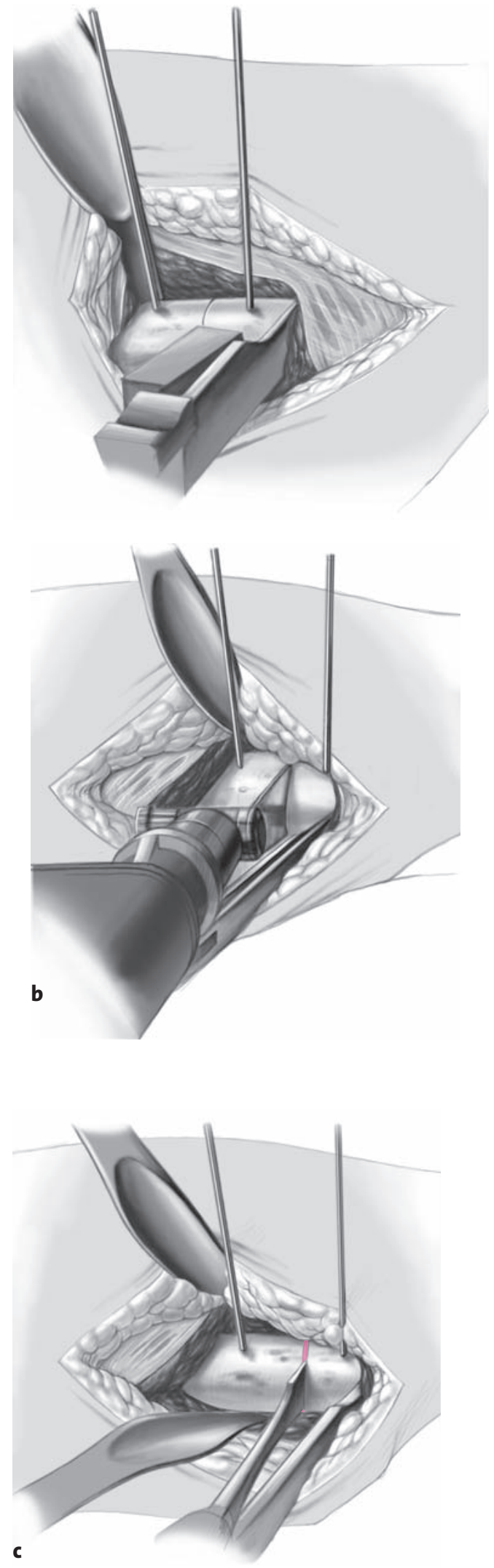


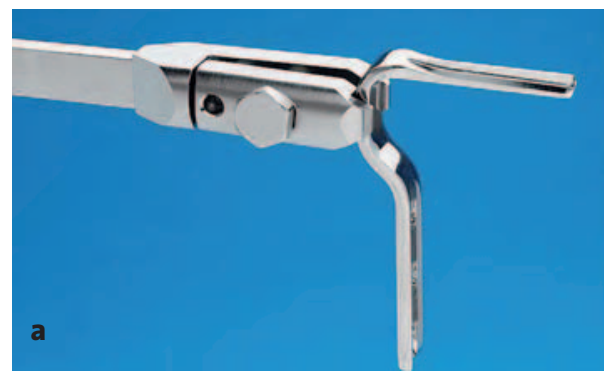

Abbildungen 13 a bis $13 \mathbf{C}$

Einführen der Platte.

Einspannen der gewählten Platte in den Inserter/Extraktor (a) und vorsichtiges Ausschlagen des kanülierten Plattensitzinstruments mit dem Schlitzhammer. Die Platte wird von Hand sorgfältig ohne Gewalt in den präformierten Schlitz eingeführt, um eine „Via falsa“ zu vermeiden (b).

Berührt das Einschlaginstrument den Knochen, wird es entfernt, und die Platte wird definitiv mit dem Impaktor eingeschlagen (c).
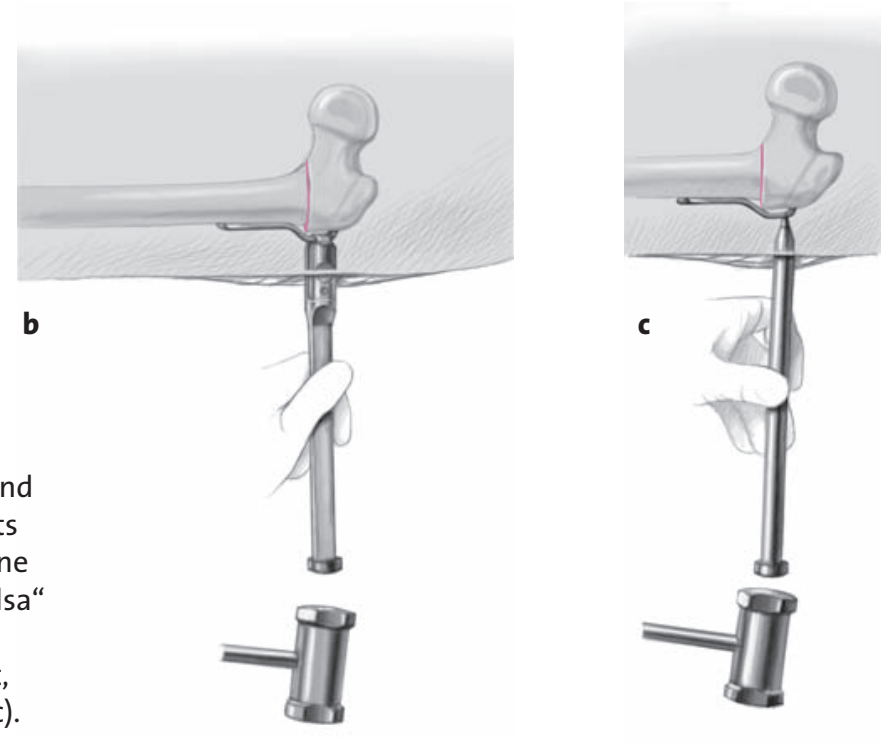

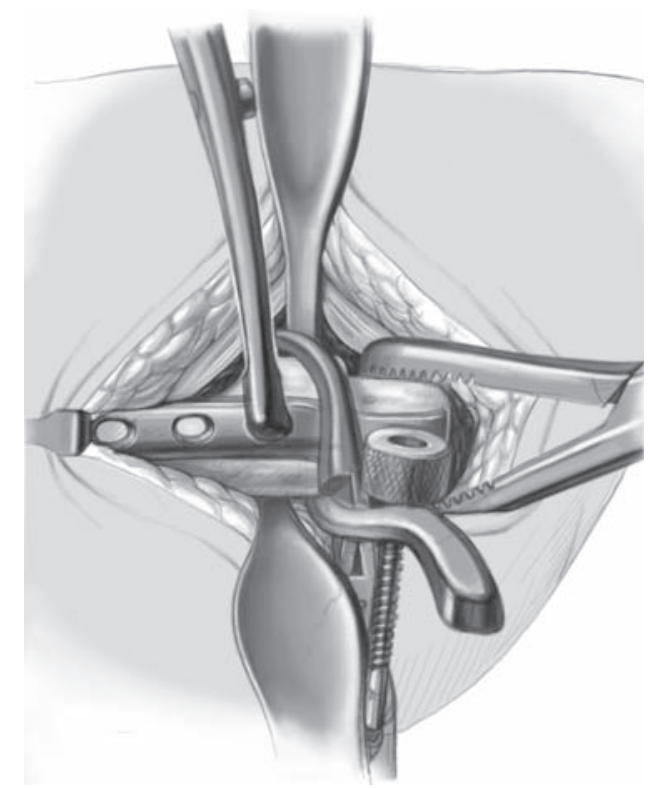

\section{Abbildung 14}

Fixierung der Platte am distalen Fragment.

Reposition des distalen Femurs an die Platte in üblicher Weise. Einstellen der Rotation. Um eine optimale interfragmentäre Kompression zu erhalten, bohren wir im mittleren Schraubenloch ohne Bohrbüchse maximal distal exzentrisch mit einem 2,5-mm-Bohrer und besetzen diese Loch.

Anschließend in gleicher Weise Bohren des distalsten Schraubenlochs; erneute Erzeugung zusätzlicher Kompression; die mittlere Schraube muss dabei etwas gelockert werden, um das Gleiten zu ermöglichen.

Setzen der proximalen Schraube, anschließend definitives Festziehen aller drei Schrauben.

\begin{abstract}
Abbildung 15
Kontrolle der Beweglichkeit und der Stabilität sowie der Lage der Platte unter dem Bildverstärker.

Wundverschluss.

Nach Ausspülen der Wunde Readaptation des abgelösten Musculus vastus lateralis mit Vicryl o. Abdecken des Muskels mit dem Bursagewebe, Vicryl 3/o. Fortlaufender Faszienverschluss mit Vicryl o. Subkutannaht und intrakutane Hautnaht mit Vicryl rapid 4/o. Üblicher Wundverband gemäß Klinikschema.
\end{abstract}




\section{LCP-Kinder-Hüftplatte (Abbildungen 16 bis 21)}

Ausgangslage: Kind $8 \mathrm{Jahre}, 28 \mathrm{~kg}$, Femur mit $160^{\circ}$ Valgus; $55^{\circ}$ Anteversion.

Operation: Varisation und Derotation linkes Femur von jeweils $30^{\circ}$.

Die Planung der Operation entspricht derjenigen des CAPOS-Systems. Gemäß der gewählten Platte muss zusätzlich der Einführungswinkels des Führungsdrahts berechnet werden (s.u.). Lagerung, Hautinzision und Zugang zum proximalen Femur sowie Lokalisation und Markierung der Trochanterphyse sind ebenfalls gleich.
Wahl des Plattentyps (s. Abbildung 5d).

In Korrelation zur Größe des Kinds und aufgrund der starken Valgusdeformität wählen wir die $110^{\circ}$-VarusLCP-Kinder-Hüftplatte, $3,5 \mathrm{~mm}$ mit $8 \mathrm{~mm}$ Offset. Entsprechend muss der Führungsdraht in einem Winkel von $140^{\circ}$ eingebracht werden $\left(110^{\circ}+30^{\circ}=140^{\circ}\right)$.

Lokalisation der Trochanterphyse.

Unter Durchleuchtung wird die Physe lokalisiert und mit einer Nadel oder einem dünnen Kirschner-Draht markiert (s. Abbildungen $9 \mathrm{~b}$ und 9c).
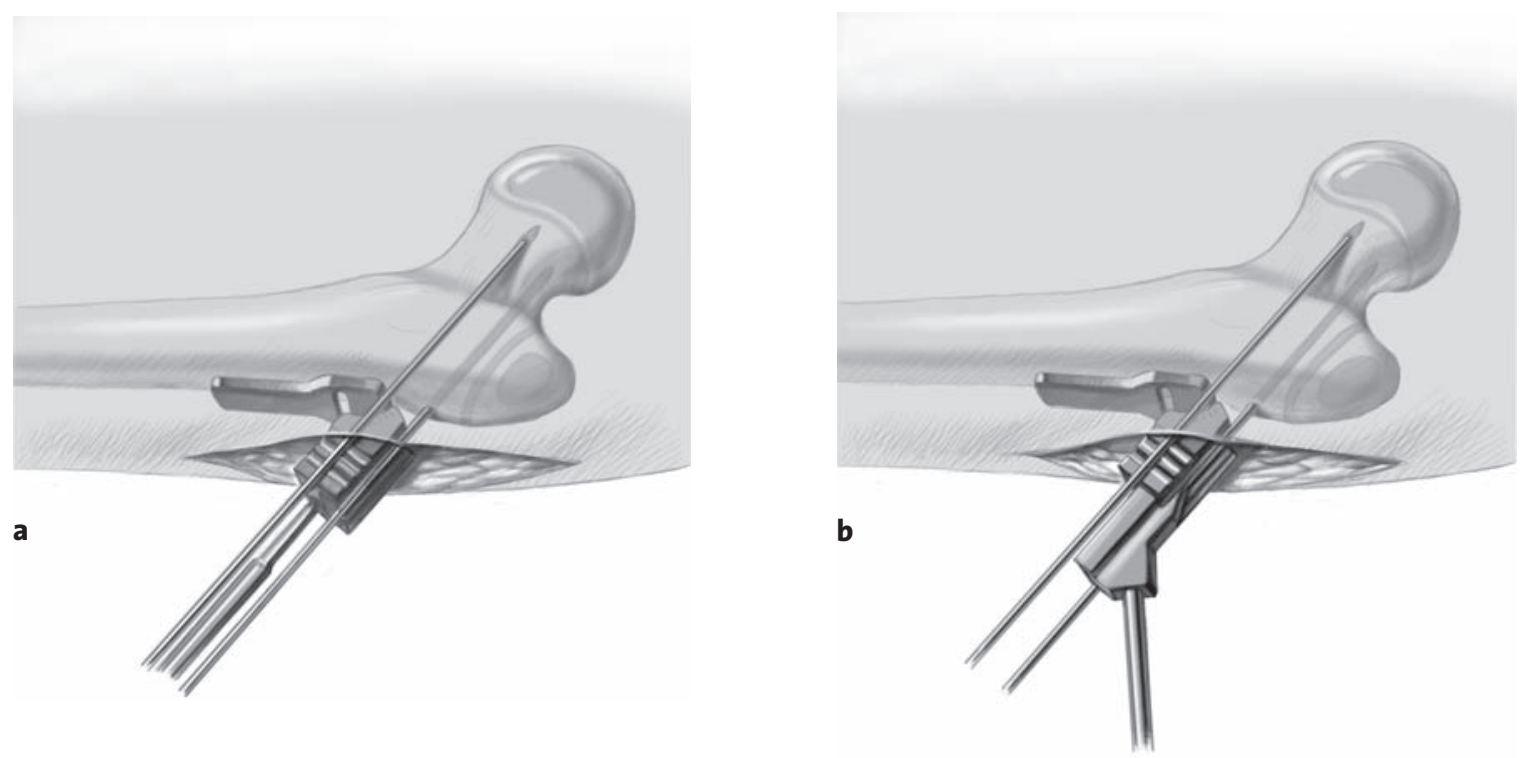

\section{Abbildungen 16a bis $16 \mathrm{c}$ \\ Festlegen der Plattenposition und Setzen des Kirschner- Führungsdrahts.}

Setzen eines 1,6-mm- oder 2,0-mm-Kirschner-Drahts über die ventrale Fläche des Schenkelhalses und leichtes Einschlagen in den Kopf. Dieser Draht markiert die Anteversion des Schenkelhalses. Aufsetzen des justierbaren Zielgeräts mit Hilfe des LCP-Hüftplatten-Führungsblocks für 3,5-mm-Schrauben, um den zentralen Führungsdraht zu setzen (Einstellung $\left.110^{\circ}+30^{\circ}=140^{\circ}\right)$. Einbohren des Führungsdrahts ca. $5 \mathrm{~mm}$ unterhalb der Fuge des großen Trochanters. Dieser muss in der Frontalebene parallel zum Anteversionsdraht liegen (a). Einbohren des 2,0-mm-Kirschner-Führungsdrahts mit Hilfe des Zielgeräts; dabei muss der Schuh des Zielgeräts am Femur aufliegen (b). Kontrolle der Lage des Kirschner-Führungsdrahts in a.p. und axialer Durchleuchtung. Im axialen Bild muss der Draht im Zentrum des Schenkelhalses liegen. Die Drahtspitze sollte die Femurkopffuge nicht perforieren (c).

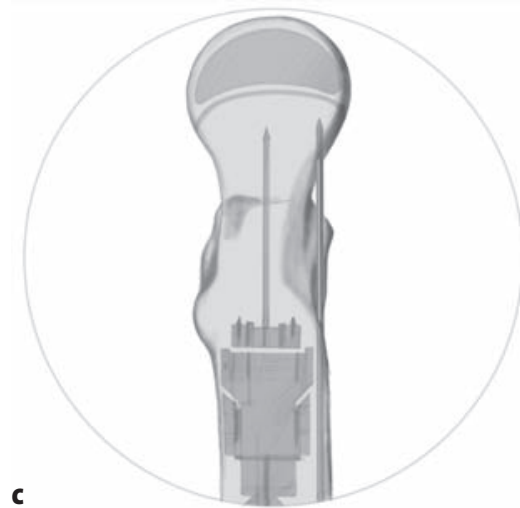




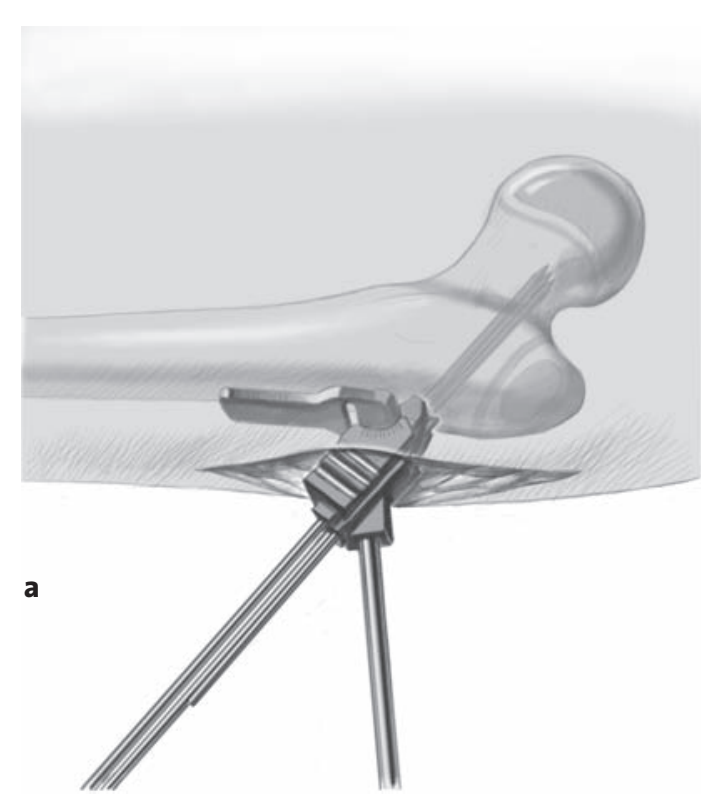

Abbildungen $\mathbf{1 7}$ a und $\mathbf{1 7} \mathbf{b}$

Setzen der beiden proximalen 2,8-mm-Kirschner-Drähte. Der korrekt platzierte justierbare Kirschner-Draht-Block garantiert zusammen mit dem dafür vorgesehenen Positionierungsblock eine genaue Platzierung der beiden 2,8-mm-KirschnerDrähte. Einbohren der beiden parallelen 2,8-mm-KirschnerDrähte in den dafür vorgesehenen Öffnungen bis knapp vor

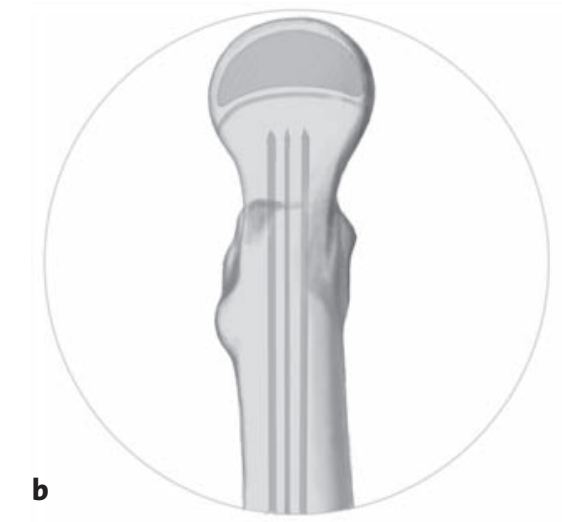

die Kopffuge (a). Es muss darauf geachtet werden, dass der freie Flügel des Blocks parallel zur Femurachse liegt, es sei denn, es soll eine Flexions- oder Extensionskorrektur erreicht werden. Entfernen der beiden Führungsblöcke. Der initiale, proximale 2,0-mm-Kirschner-Führungsdraht muss unbedingt belassen werden! Im axialen Durchleuchtungsbild kann die parallele Lage der drei Drähte gesehen und überprüft werden (b). 

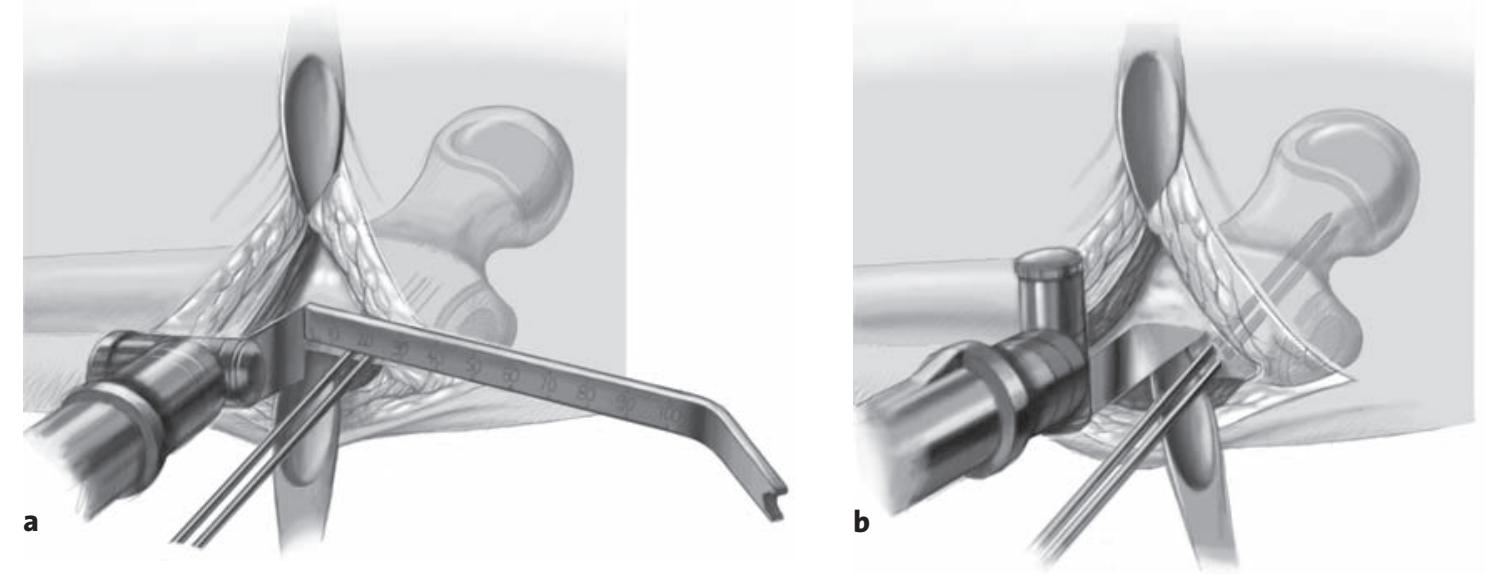

\section{Abbildungen 18a bis $\mathbf{1 8 c}$}

Festlegen der Osteotomiehöhe und der Rotation.

Die optimale Osteotomiehöhe für die 3,5-mm-Platte liegt ca. 12-15 mm distal der beiden 2,8-mm-Kirschner-Drähte (a). Diese Distanz kann mit dem dafür vorgesehenen Osteotomiedistanzmessgerät markiert werden. Setzen einer kleinen Sägemarke (b). Oberhalb und unterhalb dieser Marke kann nun je ein 2,0-mm-Kirschner-Draht so platziert werden, dass die Drähte entweder parallel zueinander liegen oder im Fall der geplanten Derotation in einem Winkel von $30^{\circ}$ zueinander eingebracht werden (c). Nach der Derotation sollten sie dann parallel stehen.

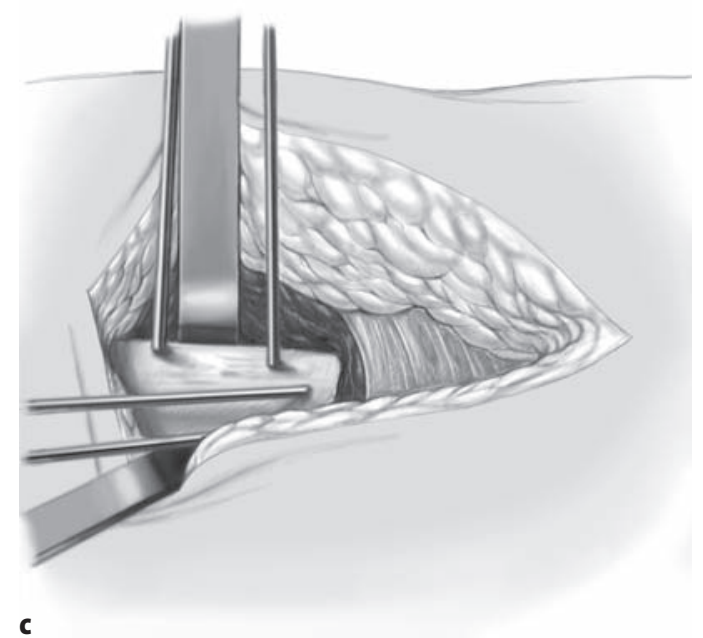

c

\section{Abbildung 19}

Setzen der Osteotomie.

Prinzipiell sollte bei dieser Technik immer rechtwinklig zur Schaftachse gesägt werden, da nur dann die sog. Kalkarschraube gesetzt werden kann.

Der Knochen wird mit einem breiten Sägeblatt durchtrennt, die Weichteile schützen wir mit zwei stumpfen, breiten Hohmann-Haken. Die Osteotomie wird an der medialen Kortikalis nicht vollständig ausgeführt, um die Gefäße zu schützen. Mit einem breiten Meißel im Osteotomiespalt und maximaler Abduktion des Beins bricht man den Knochen vollständig und löst das Periost.
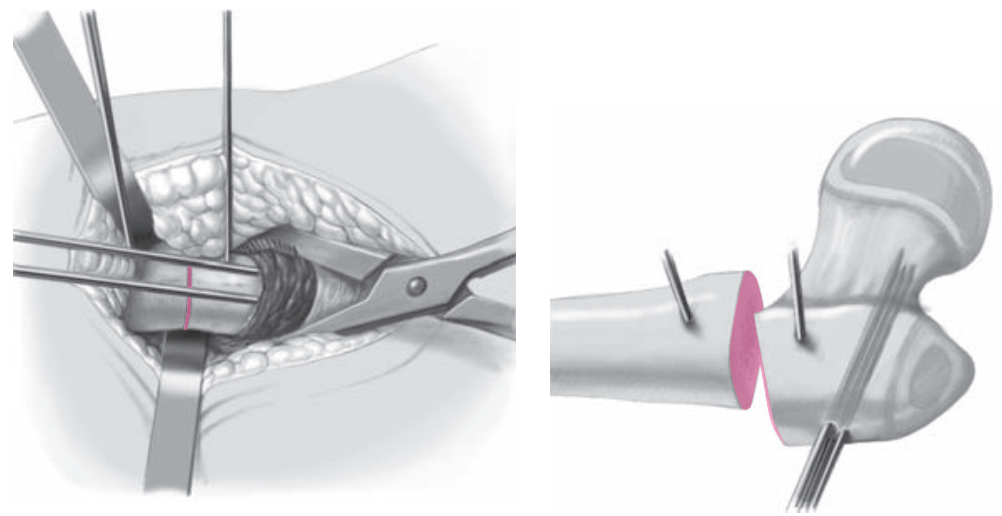


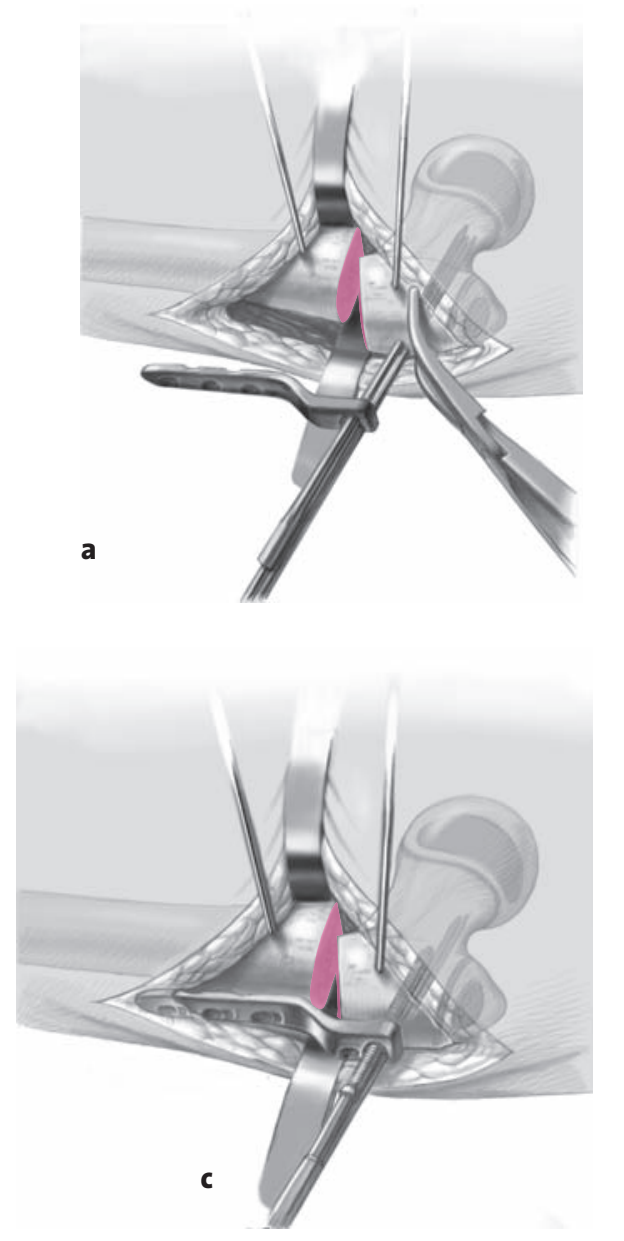

\section{Abbildungen 2oa bis 2od}

Proximale Fixierung der Platte.

In die beiden proximalen Löcher der Platte werden die Bohrbüchsen mit Gewinde eingeschraubt. Danach wird die Platte über die beiden 2,8-mm-Kirschner-Drähte geführt, und der 2,0-mm-Kirschner-Führungsdraht wird in das am proximalen Plattenende vorgesehene Loch eingefädelt (a). Die Platte wird fest gegen den Knochen gedrückt; sollte die Platte zu weit abstehen, kann sie nochmals etwas zurückgezogen werden, um einen kleineren Knochenkeil lateral am distalen Fragmentende zu entfernen.

Mit dem Messgerät, welches über die 2,8-mm-Kirschner-Drähte geschoben wird, bestimmen wir die Schraubenlänge (b). Alternativ kann ein weiterer 2,8-mm-Kirschner-Draht direkt auf
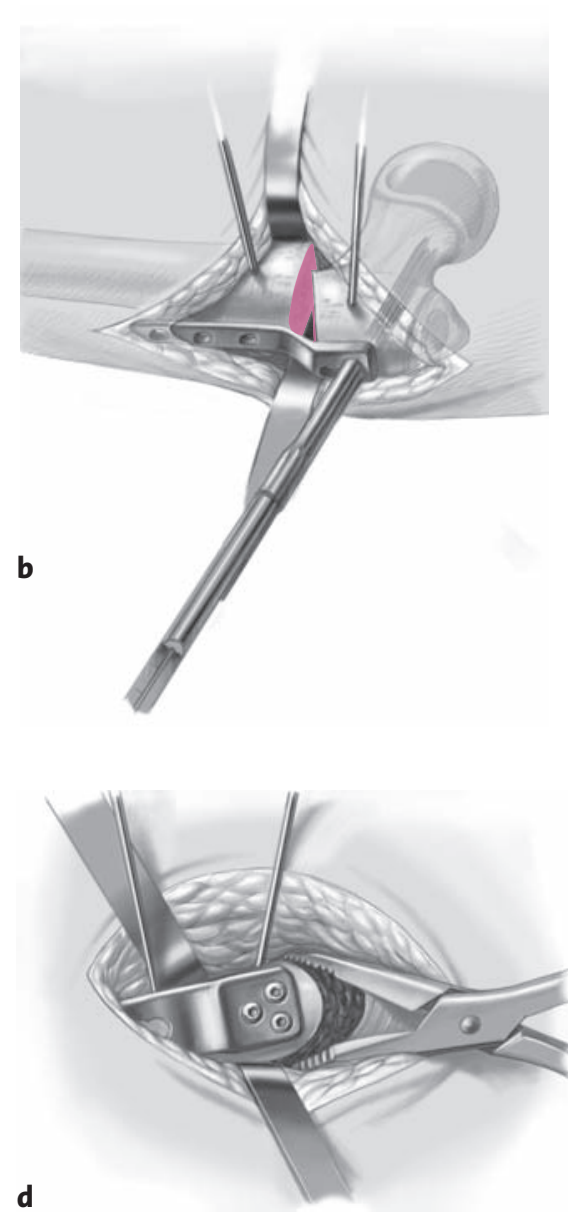

die Platte aufgesetzt werden, um die Differenz der beiden Drähte zu bestimmen.

Entfernen eines 2,8-mm-Kirschner-Drahts und der Bohrhülse sowie Eindrehen der gewählten winkelstabilen 3,5- $\mathrm{mm}$ Schrauben; endgültiges Festziehen mit dem 3,5-mmDrehmomentbegrenzer (c).

In gleicher Weise wird die zweite Schraube gesetzt. Der Führungsdraht darf während dieser Schritte nicht entfernt werden, da sonst die Rotation, also Flexion/Extension oder Neutralstellung der Platte, nicht gewährleistet ist. Nun Entfernen des Führungsdrahts. Aufschrauben der Bohrbüchse auf das distale dritte Loch und Bohren der Kalkarschraube mit dem 2,8-mm-Bohrer; Messen der Schraubenlänge und Setzen einer ebenfalls winkelstabilen Schraube (d). 


\section{Abbildungen 21a bis 21d}

Fixierung der Platte am distalen Einschrauben der beiden Bohrhülsen im proximalen und distalen Loch (a). Reposition des distalen Femurs an die Platte in üblicher Weise (b), Einstellen und Überprüfen der Rotation. Um eine optimale interfragmentäre Kompression zu erhalten, wird im mittleren Schraubenloch ohne Bohrbüchse maximal distal exzentrisch mit 2,5-mm-Bohrer monokortikal gebohrt. Einbringen einer $14 \mathrm{~mm}$ langen Kortikalisschraube; diese erzeugt eine ausreichende interfragmentäre Kompression.

Anschließend Bohren der beiden Locher für winkelstabile Schrauben, Längenmessung und Einbringen der beiden Schrauben.

Entfernen der mittleren Schraube oder Ersatz durch eine entsprechende winkelstabile Schraube; dazu muss mit der aufschraubbaren Bohrbüchse ein neues Loch gebohrt werden. Dargestellt ist der Endzustand (c).

Kontrolle der Beweglichkeit, Stabilität sowie Lage der Platte und Schrauben im Schenkelhals unter dem Bildverstärker.

Mit den beiden Medialisierungsinstrumenten

(gleiches Instrument für 3,5-mm- und 5,0-mm-Platten) kann das Femur zusätzlich nach medial geschoben werden. Dabei muss die Fixierung obligat mit winkel-

stabilen Schrauben erfolgen (d).

\section{Postoperative Behandlung CAPOS}

- Postoperative definitive Röntgenaufnahmen des Beckens a.p. und der betroffenen Hüfte nach Lauenstein. Das postoperative Ergebnis sollte unbedingt der präoperativen Planung entsprechen.

- Becken-Bein-Gipsverband je nach Situation und Kind.

- Mit Becken-Bein-Gipsverband: Hospitalisierung 4-5 Tage zum Erlernen der Pflege, Abnahme des Gipsverbands in der Regel nach 5 Wochen.

- Ohne Becken-Bein-Gipsverband: Hospitalisierung meist 6-7 Tage, um das Gehen mit Bodenkontakt an Gehstöcken zu erlernen; keine aktive Rotation, keine Flexion $>60^{\circ}$, keine aktive Abduktion; Röntgenkontrolle nach 5 Wochen. Aufgrund der Plattengeometrie und der biomechanischen Verhältnisse ist eine Vollbelastung von Beginn an nicht zu empfehlen. Es kann zu einer verzögerten Heilung und zum Stellungsverlust kommen.
- Ab der 5.-6. Woche volle Mobilisation je nach Konsolidierung möglich.

- Ab diesem Zeitpunkt Physiotherapie. Lang andauerndes Hinken bei gehfähigen Kindern ist auf eine verbliebene oder anhaltende Insuffizienz der entsprechenden Hüftmuskulatur zurückzuführen und muss auftrainiert werden.

- Doppelseitige Operationen zur gleichen Zeit führen meist zu länger dauernder Gehunfähigkeit und verlängerter Rehabilitation. Eine minimale Mobilität ist möglich.

- Sportfähigkeit nach 2-3 Monaten.

- Bei sehr mageren Kindern kann die Platte proximal sehr prominent sein und irritieren.

- Implantatentfernung frühestens nach radiologisch vollständiger Konsolidierung.

\section{LCP-Kinder-Hüftplatte}

- Postoperative definitive Röntgenaufnahmen des Beckens a.p. und der betroffenen Hüfte nach Lauen- 
stein; das postoperative Ergebnis sollte unbedingt der präoperativen Planung entsprechen.

- Ein Becken-Bein-Gipsverband ist aus Stabilitätsgründen nicht mehr notwendig, was die Pflege besonders des behinderten Kinds extrem erleichtert. Lässt sich ein Kind bezüglich Alter und Kooperation mobilisieren, ist eine partielle Belastung, d.h. mit Bodenkontakt, erlaubt. Dies ermöglicht auch die gleichzeitige Operation beider Seiten.

- Hospitalisierung meist 5-6 Tage, um das Gehen mit Bodenkontakt an Gehstöcken zu erlernen; keine aktive Rotation, keine Flexion $>60^{\circ}$, keine aktive Abduktion; Röntgenkontrolle nach 5 Wochen.

- Ab der 5.-6. Woche volle Mobilisation je nach Konsolidierung möglich.

- Aufgrund des guten anatomischen Designs der Platte sind auch bei mageren Kindern kaum Muskel- und Hautirritationen zu beobachten.

- Ab diesem Zeitpunkt kann Physiotherapie empfohlen werden. Lang andauerndes Hinken bei gehfähigen Kindern ist auf eine verbliebene oder anhaltende Insuffizienz der entsprechenden Hüftmuskulatur zurückzuführen und muss auftrainiert werden.

- Sportfähigkeit nach 2-3 Monaten.

- Implantatentfernung frühestens nach radiologisch vollständiger Konsolidierung.

\section{Fehler, Gefahren, Komplikationen}

- Falsche Planung.

- Verwechslung der Drehrichtung bei Derotation bei Operationen linke/rechte Seite; beide Male in gleiche Richtung gedreht.

- Insuffiziente Schraubenfixation.

- Infekt.

- Korrekturverlust bei falscher Technik.
- Zu lange Schrauben im Schenkelhals; Perforation in das Gelenk.

\section{Ergebnisse \\ CAPOS}

Bei korrekter Indikationsstellung und Planung sollten die Ergebnisse durchwegs gut sein. Dabei sind jedoch folgende Ergebniskriterien zu unterscheiden: Korrekturverlust, Plattenlockerung, Plattenausriss oder Plattenbruch sowie bezüglich der Indikationsstellung die bleibende Zentrierung der Hüfte. Während die ersten vier Punkte prinzipiell sehr objektiv anhand der Radiologie beurteilt werden könnten, werden sie in der Literatur höchst selten richtig erwähnt. Dementsprechend sind hier kaum Literaturangaben zu machen. Die Resultate der Kopfzentrierung werden hingegen in der Regel sehr subjektiv beurteilt, wobei nahezu nicht sichtbare Korrekturen aus subjektiver Sicht als gute Ergebnisse gewertet werden. Zudem werden vergleichbare Dezentrierungen respektive Pathologien ganz unterschiedlich korrigiert. Unter diesem Aspekt sollen hier die Ergebnisse nur bezüglich der Suffizienz der Technik erwähnt werden. In unserem Krankengut der letzten 8 Jahre haben wir lediglich eine Heilungsstörung und zwei Korrekturverluste gesehen (einmal infolge einer zu dünnen Knochenbrücke zwischen Klinge und Osteotomie, einmal wegen Lockerung der Plattenklinge). Die Korrekturen wurden unabhängig von der Grundpathologie als suffizient beurteilt.

Schlechte Ergebnisse weisen auf einen Planungsfehler hin.

Eine Ausnahme bilden Kinder mit neuromuskulären Erkrankungen, besonders Non-Walker-Patienten. Diese Kinder können gelegentlich unerwartete Reaktionen auf die neue Stellung zeigen, und oft ist die Rehabilita-

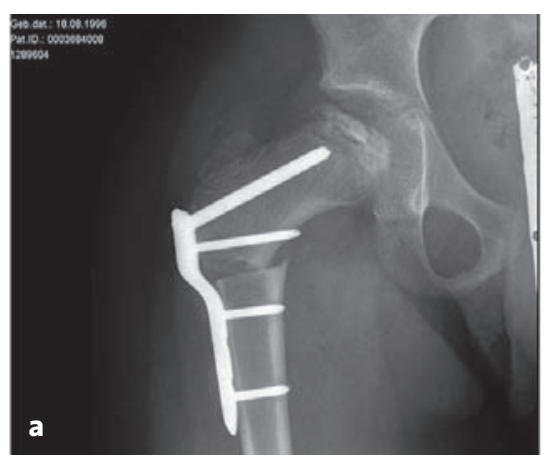

Abbildungen 22a bis 22c

Die postoperativen Röntgenbilder zeigen eine suffiziente Korrektur sowie korrekte Lage der Platte nach Varisationsosteotomie bei Morbus Perthes.
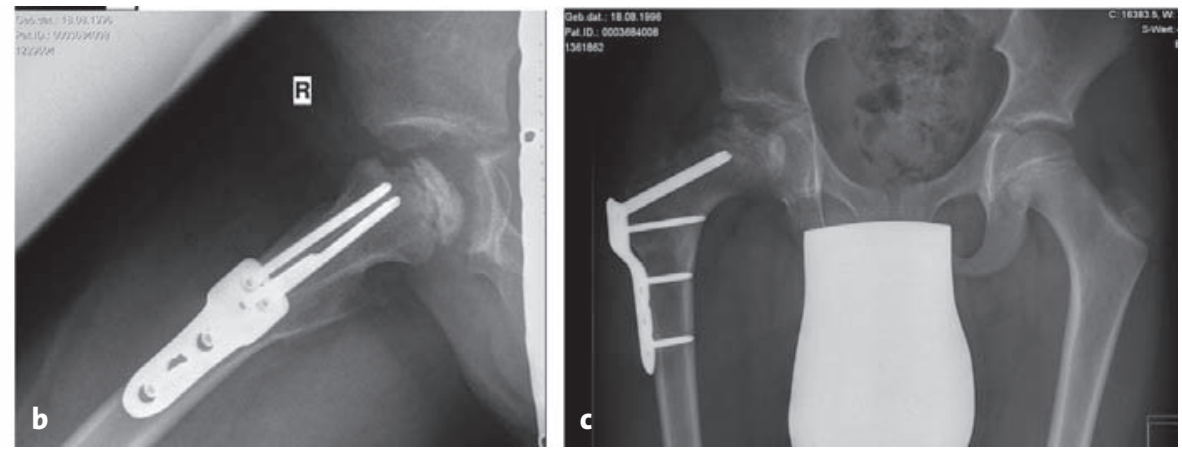

a) A.p. Ansicht.

b) Axiale Ansicht.

c) Vollständige Heilung ohne großen Kallus 6 Monate postoperativ. 
tion erschwert und verlängert, was zur Unzufriedenheit bei den betreuenden Personen führt. Hier ist Geduld angesagt.

Vorzeitige Korrekturen von Valgusdeformitäten bei kleineren Kindern können nach Entfernung der Platten wiederum in eine Valgusstellung zurückkehren.

\section{LCP-Kinder-Hüftplatte (Abbildungen 22a bis 22c)}

Die ersten Ergebnisse sind mehr als befriedigend, sogar vielversprechend und vermögen die in dieses Implantat gesetzten Ziele in angemessener Weise zu erfüllen. Da es sich um eine neues Implantat handelt, liegen noch keine entsprechenden umfassenden Studien vor, doch die im Rahmen der Einführung geforderten, noch nicht publizierten Ergebnisse von vier international renommierten Kliniken zeigen keine Probleme bezüglich Heilung, Korrekturverlust oder Implantat. Wie auch mit der CAPOS-Technik ist die Indikationsstellung sehr breit, und entsprechend sind die angestrebten Korrekturen individuell sehr unterschiedlich.

Ein erster indirekter Hinweis auf die exzellente Stabilität ist die sog. direkte Osteotomieheilung, d.h., der sonst bei der Winkelplatte sichtbare voluminöse Kallus ist nicht zu verzeichnen, sondern es kommt zur primären Konsolidierung. Aufgrund des anatomischen Designs bildet sich auch keine störende Pseudobursa über dem Plattenknie, was bei den oft sehr mageren, spastischen Kindern von besonderem Vorteil ist.

\section{Literatur}

1. Hefti F, et al. Kinderorthopädie in der Praxis. Berlin-Heidelberg-New York: Springer, 1998.

2. Morrissy RT, Weinstein SL. Atlas of pediatric orthopedic surgery. Philadelphia: Williams \& Wilkins, 2001

3. Müller ME. Die hüftnahen Femurosteotomien, 2. Aufl. Stuttgart: Thieme, 1971.

4. Müller ME. Intertrochanteric osteotomy: indication, preoperative planning technique. In: Schatzker J, ed. The intertrochanteric osteotomy. Berlin-Heidelberg-New York: Springer, 1984:25-66.

5. Müller ME, Allgöwer $M$, Schneider R, et al. AO manual of internal fixation, 3rd edn. Berlin-Heidelberg-New York: Springer, 1991.

\section{Korrespondenzanschrift}

Dr. Theddy Slongo Leitender Arzt für Kindertraumatologie und Kinderorthopädie Abteilung für Pädiatrische Chirurgie Universitätskinderklinik Inselspital 3010 Bern Schweiz Telefon (+41/31) 632-9293, Fax -9292

E-Mail:theddy.slongo@insel.ch 